\title{
ULTRAFILTERS, FINITE COPRODUCTS AND LOCALLY CONNECTED CLASSIFYING TOPOSES
}

\author{
RICHARD GARNER
}

\begin{abstract}
We prove a single category-theoretic result encapsulating the notions of ultrafilters, ultrapower, ultraproduct, tensor product of ultrafilters, the Rudin-Kiesler partial ordering on ultrafilters, and Blass's category of ultrafilters $\mathcal{U F}$. The result in its most basic form states that the category FC(Set, Set) of finite-coproduct-preserving endofunctors of Set is equivalent

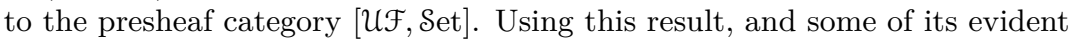
generalisations, we re-find in a natural manner the important model-theoretic realisation relation between $n$-types and $n$-tuples of model elements; and draw connections with Makkai and Lurie's work on conceptual completeness for first-order logic via ultracategories.

As a further application of our main result, we use it to describe a first-order analogue of Jónsson and Tarski's canonical extension. Canonical extension is an algebraic formulation of the link between Lindenbaum-Tarski and Kripke semantics for intuitionistic and modal logic, and extending it to first-order logic has precedent in the topos of types construction studied by Joyal, Reyes, Makkai, Pitts, Coumans and others. Here, we study the closely related, but distinct, construction of the locally connected classifying topos of a first-order theory. The existence of this is known from work of Funk, but the description is inexplicit; ours, by contrast, is quite concrete.
\end{abstract}

\section{INTRODUCTION}

Ultrafilters are important in many areas of mathematics, from Ramsey theory, to topological dynamics, to universal algebra; see [7] for an overview. Around the notion of ultrafilter is a circle of associated concepts: the ultrapower of a set by an ultrafilter, or more generally, the ultraproduct of a family of sets [17]; the tensor product of ultrafilters [30] and the more general indexed sum; and the Rudin-Keisler partial ordering on ultrafilters, first written down by Blass in [5] and immediately enhanced to a category of ultrafilters.

Of course, ultrafilters are particularly important in model theory. One aspect of this is that complete $n$-types of a first-order theory $\mathbb{T}$ are precisely ultrafilters on the Lindenbaum-Tarski algebra of $\mathbb{T}$ extended by $n$ new constants; in particular, each $n$-tuple of elements of a $\mathbb{T}$-model has an associated complete $n$-type, and this realisation relation between $n$-tuples and $n$-types is central to many questions in model theory.

Date: 3rd June 2020.

2000 Mathematics Subject Classification. Primary:

The support of Australian Research Council grants DP160101519 and FT160100393 is gratefully acknowledged. Thanks also to the referees and the editor for helpful and constructive suggestions, which have improved the paper significantly. 
Ultrafilters also provide the link between the semantics for modal logic valued in modal algebras (Boolean algebras with operators), and the Kripke semantics; indeed, the canonical Kripke model associated to a modal algebra $B$ takes its frame of possible worlds to comprise exactly the ultrafilters on $B$. A similar relation holds between the Lindenbaum-Tarski and Kripke semantics for intuitionistic propositional logic $[46, \S 2.6]$; and these relations have been expressed algebraically via Jónsson and Tarski's notion of canonical extension [27, 21]. Canonical extension has been generalised from propositional to first-order logic via the topos of types construction studied by Joyal and Reyes [29], Makkai [38] and Pitts [44] — and again, ultrafilters play a key role.

Another key use of ultrafilters in model theory is via Los' theorem that the models of a first-order theory $\mathbb{T}$ are closed under ultraproducts. This is useful in its own right, for example in constructing saturated models, but has also been exploited more structurally by Makkai [40] and Lurie [35]: they prove a "conceptual completeness" theorem which can reconstruct a first-order theory $\mathbb{T}$ (or at least, its completion $\mathbb{T}^{\text {eq }}$ under elimination of imaginaries) from the category of models and elementary embeddings, together with the ultraproduct structure on this category.

The objective of this paper is to describe a single category-theoretic result from which the notions of ultrafilter, ultrapower, tensor product of ultrafilters, and Blass's category UF of ultrafilters, together with their interrelations, all flow naturally - and which, with only a little more effort, is able to speak towards the applications of ultrafilters in model theory described above. This result, which is Theorem 13 and Corollary 14 below, may be stated as follows:

Theorem. The category FC(Set, Set) of finite-coproduct-preserving endofunctors of Set is equivalent to the category [UF, Set] of functors on Blass' category [5] of ultrafilters $\mathcal{U F}$. Under this equivalence, the ultrapower functor $(-)^{\mathfrak{U}}$ corresponds to the representable functor at the ultrafilter $\mathcal{U}$.

This builds on Börger's characterisation [9] of the functor $\beta$ : Set $\rightarrow$ Set, which sends a set $X$ to its set of ultrafilters, as the terminal finite-coproduct-preserving endofunctor of Set. (Though it will not play any role here, we should also mention [32]'s different characterisation of $\beta$ as the codensity monad of the inclusion functor FinSet $\hookrightarrow$ Set; see [34] for a modern account.)

One way of seeing our result is that once we know what a finite coproductpreserving endofunctor of Set is, everything else is forced. The ultrapower endofunctors of Set arise as the projective indecomposable objects in FC(Set, Set), and the full subcategory they span is equivalent to $\mathcal{U F}^{\mathrm{op}}$. Moreover, as we will see in Proposition 16, the composition monoidal structure on FC (Set, Set) restricts to this subcategory, and in this way recovers the tensor product of ultrafilters.

One thing this theorem does not capture is the notion of ultraproduct. For this, we require a generalisation of the theorem dealing with ultrafilters not on sets, but on objects of a category $\mathcal{C}$ which is extensive [12], meaning that it has well-behaved finite coproducts. In this context, an ultrafilter on $X \in \mathcal{C}$ can be defined as an ultrafilter on the Boolean algebra of coproduct summands of $X$, 
giving rise to a category $\mathcal{U F}_{\mathcal{C}}$ generalising Blass' $\mathcal{U F}$. We now obtain the following natural generalisation of our main theorem, to be proved as Theorem 22:

Theorem. Let $\mathcal{C}$ be extensive. The category $\mathrm{FC}(\mathcal{C}$, Set) of finite-coproduct-preserving

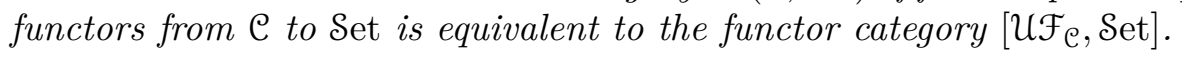

As we will see in Section 4.2, we may recapture ultraproducts from this theorem by taking $\mathcal{C}=\operatorname{Set}^{X}$, yielding an equivalence $\left[\mathcal{U} \mathcal{F}_{\operatorname{Set}^{X}}, \mathcal{S e t}\right] \simeq \mathrm{FC}\left(\operatorname{Set}^{X}\right.$, Set $)$; now the ultraproduct functors $\Pi_{\mathcal{U}}: \mathcal{S e t}^{X} \rightarrow \mathcal{S}$ et correspond under this equivalence to suitable representable functors in [ $\mathcal{\mathcal { F } _ { \text { Set } }}{ }$, Set]

A second application, described in Section 4.3, takes $\mathcal{C}$ to be the classifying Boolean pretopos of a theory $\mathbb{T}$ of classical first-order logic, which plays the same role for a first-order theory as does the Lindenbaum-Tarski algebra of a propositional theory. In this case, ultrafilters on $A \in \mathcal{C}$ correspond to modeltheoretic types in context $A$, and our result allows us to reconstruct a categorical treatment of these [38]. Indeed, by the classifying property of $\mathcal{C}$, models of $\mathbb{T}$ correspond to pretopos morphisms $\mathcal{C} \rightarrow$ Set. As pretopos morphisms preserve finite coproducts, the theorem thereby associates to each $\mathbb{T}$-model $M$ a functor $\mathcal{U} \mathcal{F}_{e} \rightarrow$ Set-whose values pick out the sets of $M$-elements that realise each type.

Our main theorem can be generalised further by varying the codomain category as well as the domain category. Recall that a Grothendieck topos is the category of sheaves on a small site, and that a Grothendieck topos $\mathcal{E}$ is locally connected when the left adjoint $\Delta:$ Set $\rightarrow \mathcal{E}$ of its global sections functor $\Gamma=\mathcal{E}(1,-): \mathcal{E} \rightarrow$ Set has a further left adjoint $\pi_{0}: \mathcal{E} \rightarrow$ Set. The second generalisation of our main theorem, to be proved as Theorem 26 below, is now:

Theorem. Let $\mathcal{C}$ be extensive and $\mathcal{E}$ a locally connected Grothendieck topos. The category $\mathrm{FC}(\mathcal{C}, \mathcal{E})$ of finite-coproduct-preserving functors from $\mathcal{C}$ to $\mathcal{E}$ is equivalent to the functor category $\left[\mathcal{U F}_{\mathcal{C}}, \mathcal{E}\right]$.

One application of this theorem, described in Section 5.2, allows us to reconstruct the indexed sum of ultrafilters. For any sets $X$ and $Y$, our theorem yields an equivalence $\mathrm{FC}\left(\mathcal{S e t}^{X}, \mathcal{S e t}^{Y}\right) \simeq\left[\mathcal{U F}_{\mathcal{S e t}^{X}}, \mathcal{S e t}^{Y}\right]$, and we define a generalised ultraproduct functor $\operatorname{Set}^{X} \rightarrow \operatorname{Set}^{Y}$ to be one that corresponds under this equivalence to a pointwise representable functor $U \mathcal{F}_{\operatorname{Set}^{X}} \rightarrow \mathcal{S e t}^{Y}$. Such functors have a representation as ultraspans: that is, as diagrams

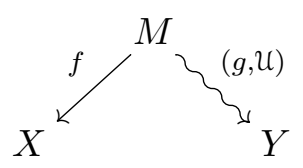

with left leg a function $f$ and right leg a function $g$ endowed with an ultrafilter $\mathcal{U}_{y}$ on each fibre $g^{-1} y$. Moreover, it turns out that generalised ultraproduct functors are closed under composition, so inducing a composition law on ultraspans (1.1) which encodes perfectly the indexed sum of ultrafilters.

Another potential application of the above theorem, sketched in Remark 32, is to Makkai's ultracategories [40]. An ultracategory is a category $\mathcal{C}$ endowed with abstract ultraproduct functors $\Pi_{\mathfrak{U}}: \mathcal{C}^{X} \rightarrow \mathcal{C}$ together with interpretations for any "definable map between ultraproducts" - the ultramorphisms of [40]. The 
key example of an ultracategory is the category of models of a coherent theory $\mathbb{T}$ in intuitionistic first-order logic, and [40]'s main result shows that, to within Morita equivalence, $\mathbb{T}$ can be reconstructed from its ultracategory of models.

We expect to relate ultracategories to our main result via the machinery of enriched categories [31, 47]. We have calculated far enough to convince ourselves that categories endowed with abstract ultraproduct functors can be identified with certain categories enriched over the bicategory FC $_{\text {Set }}$ of finite-coproductpreserving functors between powers of Set which admit certain copowers (a kind of enriched colimit). The key point is that, in proving this, we exploit the equivalences $\mathrm{FC}\left(\mathcal{S e t}^{X}, \operatorname{Set}^{Y}\right) \simeq\left[\mathcal{U} \mathcal{F}_{\operatorname{Set}^{X}}\right.$, $\left.\operatorname{Set}^{Y}\right]$ established above. We will develop this line of thought further in future work.

Our final main result exploits the preceding theorems to construct the locally connected classifying topos of a suitable pretopos $\mathcal{C}$. This construction is similar to the toposes of types mentioned above [29, 38, 44], in that it provides a first-order analogue to the operation of canonical extension [27, 21] on propositional theories. The existence of locally connected classifying toposes follows from [18]; however, the existence proof given there is somewhat inexplicit. We will improve on this by showing that any small pretopos satisfying the De Morgan property (recalled in Definition 38 below) has a locally connected classifying topos given by the topos of sheaves on $\mathcal{U F}_{\mathcal{C}}$ for a certain Grothendieck topology, related to one found in [29]. Our final main result, proved as Theorem 42 below, is thus:

Theorem. Let $\mathcal{C}$ be a small De Morgan pretopos. The topos $\mathrm{Sh}\left(\mathcal{U F}_{\mathcal{C}}\right)$ is a locally connected classifying topos for $\mathrm{C}$, and is itself De Morgan.

While we discuss briefly the similarities and differences between this construction, and the various toposes of types in the literature, we will, once again, leave a more detailed comparison to future work.

\section{BACKGROUND}

2.1. Ultrafilters, ultraproducts and ultrapowers. In this section, we recall the notions that our main theorem is designed to capture and their interrelations with each other. Before starting on this, we first establish some notational conventions for indexed families which will be used throughout the paper.

Definition 1. Let $Y=(Y(x) \mid x \in X)$ be an $X$-indexed family of sets. We write $(\Sigma x \in X) Y(x)$ or more briefly $X . Y$ for the indexed sum of this family, that is, the set of pairs $\{(x, y): x \in X, y \in Y(x)\}$. We write $\pi_{Y}: X . Y \rightarrow X$ for the first projection map, and call this map the display family associated to $Y$. We also write $(\Pi x \in X) Y(x)$ for the indexed product of the $Y(x)$ 's: that is, the set of functions $f: X \rightarrow X . Y$ which are sections of $\pi_{Y}: X . Y \rightarrow X$.

More generally, a display family over $X$ is any function $\pi: E \rightarrow X$, and the $X$-indexed family associated to $\pi$ is the family of fibres $\left(\pi^{-1}(x) \mid x \in X\right)$. As is well known, the passage between $X$-indexed families and display families over $X$ underlies an equivalence of categories

$$
\operatorname{Set}^{X} \simeq \operatorname{Set} / X
$$

This equivalence and its generalisations will play an important role in this paper. 
Definition 2. An ultrafilter on a set $X$ is a Boolean algebra homomorphism $u: \mathcal{P} X \rightarrow 2$. Most often, we describe $u$ by specifying the subset $\mathcal{U}=u^{-1}(\top)$ of $\mathcal{P} X$; so an ultrafilter is equally a collection $\mathcal{U}$ of subsets of $X$ such that:

(i) $X \in \mathcal{U}$, and $U \cap V \in \mathcal{U} \Longleftrightarrow(U \in \mathcal{U}$ and $V \in \mathcal{U})$;

(ii) $\perp \notin \mathcal{U}$, and $U \cup V \in \mathcal{U} \Longleftrightarrow(U \in \mathcal{U}$ or $V \in \mathcal{U})$.

Equivalently, we may replace condition (ii) with:

(ii) ${ }^{\prime} U \in \mathcal{U} \Longleftrightarrow X \backslash U \notin \mathcal{U}$.

We write $\beta X$ for the set of ultrafilters on the set $X$.

The principal ultrafilter at $x \in X$ is $\uparrow x=\{U \subseteq X: x \in U\}$. These are the only ultrafilters we can write down explicitly; indeed, the existence of non-principal ultrafilters is a choice principle, slightly weaker than the axiom of choice [23].

It is often useful to view ultrafilters as generalised quantifiers. Given a predicate $\varphi(x)$ depending on $x \in X$ and an ultrafilter $\mathcal{U}$ on $X$, we write $(\forall u x \in X) \varphi(x)$ to indicate that $\{x \in X: \varphi(x)\} \in \mathcal{U}$ and say that "for U-almost all $x, \varphi(x)$ holds".

Definition 3. Let $\mathcal{U} \in \beta X$. If $Y$ is a set, then the ultrapower $Y^{\mathcal{U}}$ is the set of $=\mathfrak{u}$-equivalence classes of partial functions $X \rightarrow Y$ defined on a set in $\mathcal{U}$, where

$$
f=u g \quad \text { iff } \quad(\forall u x \in X) f(x) \equiv g(x) .
$$

Here we write $f(x) \equiv g(x)$ to mean " $f$ and $g$ are defined at $x$ and are equal".

More generally, if $Y$ is an $X$-indexed family of sets, then the ultraproduct $\left(\Pi_{\mathcal{U}} x \in X\right) Y(x)$ is the set of $=\mathfrak{u}$-equivalence classes of partial sections, defined on a set in $\mathcal{U}$, of $\pi_{Y}: X . Y \rightarrow X$. Note that $Y^{\mathfrak{U}}=\left(\Pi_{\mathfrak{U}} x \in X\right) Y$.

We can take a topological view on ultraproducts. Given an $X$-indexed family $Y$, we can view the projection $(\Sigma x \in X) Y(x) \rightarrow X$ as a local homeomorphism between discrete spaces. Pushing this forward along the embedding $X \rightarrow \beta X$ of $X$ into its Stone-Cěch compactification yields a local homeomorphism over $\beta X$, whose fibre over an ultrafilter $\mathcal{U} \in \beta X$ is the ultraproduct $\left(\Pi_{\mathcal{U}} x \in X\right) Y(x)$.

The explicit formula for pushforward of local homeomorphisms - in terms of germs of local sections - yields the following reformulation of ultrapowers and ultraproducts in terms of colimits; herein, we view $\mathcal{U}$ as a poset ordered by inclusion:

$$
\begin{aligned}
Y^{\mathfrak{U}} & =\operatorname{colim}_{U \in \mathcal{U}} Y^{U} \\
\left(\Pi_{\mathcal{U}} x \in X\right) Y(x) & =\operatorname{colim}_{U \in \mathcal{U}}(\Pi x \in U) Y(x) .
\end{aligned}
$$

This description makes it clear that ultraproduct and ultrapower are functors $(-)^{\mathcal{U}}:$ Set $\rightarrow$ Set and $\Pi_{\mathcal{U}}:$ Set $^{X} \rightarrow$ Set respectively.

2.2. The category of ultrafilters. Given ultrafilters $\mathcal{U}$ on $X$ and $\mathcal{V}$ on $Y$, we say that $f: X \rightarrow Y$ is continuous if $V \in \mathcal{V}$ implies $f^{-1}(V) \in \mathcal{U}$. By axiom (ii)' and the fact that $f^{-1}$ preserves complements, this is equally the condition that

$$
V \in \mathcal{V} \Longleftrightarrow f^{-1}(V) \in \mathcal{U} \text {. }
$$

The continuous maps play an important role in two natural categories of ultrafilters, originally defined in $[30,33]$ in the more general context of filters. 
Definition 4. The category $\mathcal{U} \mathcal{E}$ of ultrafilters has pairs $(X \in \mathcal{S}$ et, $\mathcal{U} \in \beta X)$ as objects, and as maps $(X, \mathcal{U}) \rightarrow(Y, \mathcal{V})$ the continuous maps $X \rightarrow Y$. The category $\mathcal{U F}$ of ultrafilters has the same objects, and as morphisms $(X, \mathcal{U}) \rightarrow(Y, \mathcal{V})$ the

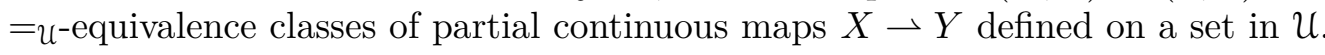

Our naming reflects that $\mathcal{U F}$ is the "good" category of ultrafilters and $\mathcal{U} \mathcal{E}$ just a preliminary step to get there; for indeed, $\mathcal{U F}$ arises by inverting the class $\mathcal{M}$ of continuous injections in $\mathcal{U E}$. The proof of this fact given below mirrors that given in [6, Theorem 16] for the category of filters; in its statement, $\iota: \mathcal{U E} \rightarrow \mathcal{U F}$

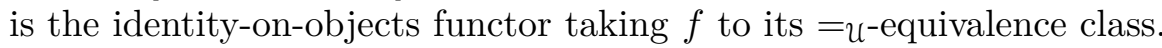

Proposition 5. $\iota: U \mathcal{E} \rightarrow U \mathcal{F}$ exhibits $U \mathcal{F}$ as $U \mathcal{E}\left[\mathcal{M}^{-1}\right]$.

Proof. Each map in $\mathcal{M}$ factors as an isomorphism followed by a continuous subset inclusion; whence $\mathcal{U} \mathcal{E}\left[\mathcal{M}^{-1}\right]=\mathcal{U} \mathcal{E}\left[\mathcal{J}^{-1}\right]$ where $\mathcal{J}$ is the class of all continuous subset inclusions in $\mathcal{U} \mathcal{E}$. It is easy to see that any map in $\mathcal{J}$ is of the form

$$
m_{W Y}:\left(W,\left.\mathcal{V}\right|_{W}\right) \hookrightarrow(Y, \mathcal{V})
$$

where $W \in \mathcal{V}$ and $\left.\mathcal{V}\right|_{W}=\{U \subseteq W: U \in \mathcal{V}\}$. Such maps are stable under composition and contain the identities. Moreover, given a map (2.5) and $f:(X, \mathcal{U}) \rightarrow(Y, \mathcal{V})$ in $\mathcal{U} \mathcal{E}$, we have a commuting square (in fact a pullback) in $\mathcal{U E}$ of the form

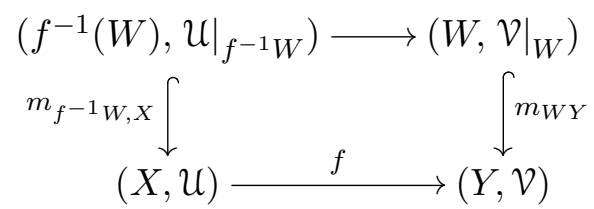

since $f^{-1}(W) \in \mathcal{U}$ by continuity of $f$. So $\mathcal{J}$ satisfies the first three of the four axioms for a calculus of right fractions [19], and satisfies the final one trivially since it is a class of monomorphisms. We may thus describe the localisation $\mathcal{U} \mathcal{E}\left[\mathcal{J}^{-1}\right]$ as follows. Objects are those of $\mathcal{U} \mathcal{E}$, and maps $(X, \mathcal{U}) \rightarrow(Y, \mathcal{V})$ are spans in $\mathcal{U} \mathcal{E}$ as to the left below, with two such spans being identified if they can be completed to a commuting diagram as to the right.
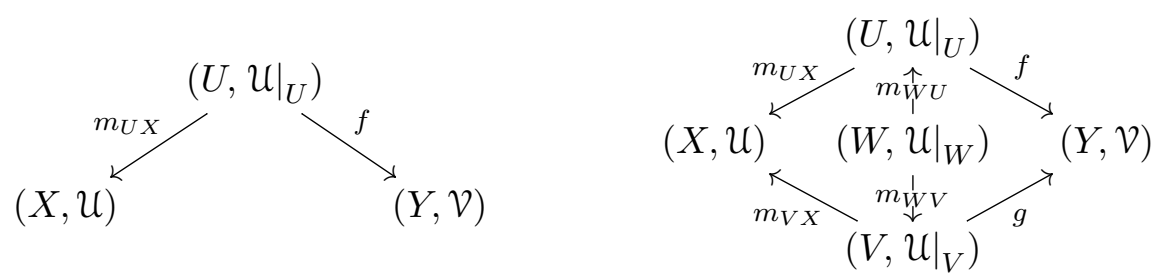

Clearly these maps correspond to $=\mathfrak{u}$-equivalence classes of partial continuous functions; moreover, under this identification, the identity-on-objects functor $\mathcal{U} \mathcal{E} \rightarrow \mathcal{U} \mathcal{E}\left[\mathcal{J}^{-1}\right]$ sends $f$ to $(1, f)$, whence $\mathcal{U} \mathcal{F} \cong \mathcal{U} \mathcal{E}\left[\mathcal{J}^{-1}\right]$ under $\mathcal{U} \mathcal{E}$ as desired.

2.3. Tensor product and indexed sum of ultrafilters. The tensor product of ultrafilters is sometimes called the product. It is most easily expressed in terms of generalised quantifiers. 
Definition 6. Let $\mathcal{U}$ and $\mathcal{V}$ be ultrafilters on $X$ and $Y$. The tensor product $\mathcal{U} \otimes \mathcal{V}$ is the unique ultrafilter on $X \times Y$ which for all predicates $\varphi$ on $X \times Y$ satisfies:

$$
(\forall \mathfrak{u} \otimes \mathcal{V}(x, y) \in X \times Y) \varphi(x, y) \quad \Longleftrightarrow \quad\left(\forall_{u} x \in X\right)\left(\forall_{\mathcal{v}} y \in Y\right) \varphi(x, y) .
$$

Instantiating $\varphi$ at the characteristic predicates of subsets $A \subseteq X \times Y$ yields the following explicit formula, wherein we write $x^{*} A$ for $\{y \in Y:(x, y) \in A\}$ :

$$
\mathcal{U} \otimes \mathcal{V}=\left\{A \subseteq X \times Y:\left\{x \in X: x^{*} A \in \mathcal{V}\right\} \in \mathcal{U}\right\}
$$

Using this formula, we see that if $f:(X, \mathcal{U}) \rightarrow\left(X^{\prime}, \mathcal{U}^{\prime}\right)$ and $g:(Y, \mathcal{V}) \rightarrow\left(Y^{\prime}, \mathcal{V}^{\prime}\right)$ in $\mathcal{U} \mathcal{E}$ then also $f \times g:(X \times Y, \mathcal{U} \otimes \mathcal{V}) \rightarrow\left(X^{\prime} \times Y^{\prime}, \mathcal{U}^{\prime} \otimes \mathcal{V}^{\prime}\right)$. So tensor product of ultrafilters gives a monoidal structure on $\mathcal{U} \mathcal{E}$, with as unit the unique ultrafilter on the one-element set. Since maps in $\mathcal{M}$ are closed under the binary tensor, this monoidal structure descends along $\iota$ to one on $\mathcal{U F}$.

The following result, which is a special case of [17, Theorem 1.10], describes the interaction of the tensor product with ultrapowers and ultraproducts.

Proposition 7. Given $\mathcal{U} \in \beta X$ and $\mathcal{V} \in \beta Y$ and an $X \times Y$-indexed family of sets $Z$, currying of functions induces an isomorphism of ultraproducts

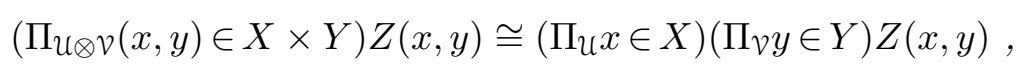

giving, when $Z$ is a constant family, isomorphisms $Z^{\mathcal{U} \otimes \mathcal{V}} \cong\left(Z^{\mathcal{V}}\right)^{\mathcal{U}}$.

A more general construction on ultrafilters is that of indexed sum.

Definition 8. Let $\mathcal{U}$ be an ultrafilter on $X$ and, for each $x \in X$, let $\mathcal{V}(x)$ be an ultrafilter on $Y(x)$. The indexed sum $\left(\Sigma_{\mathcal{U}} x \in X\right) \mathcal{V}(x)$ or $\mathcal{U} . \mathcal{V}$ is the unique ultrafilter on $(\Sigma x \in X) Y(x)=X . Y$ which for all predicates $\varphi$ on $X . Y$ satisfies

$$
(\forall u . v(x, y) \in X . Y) \varphi(x, y) \Longleftrightarrow(\forall u x \in X)\left(\forall_{\mathcal{v}(x)} y \in Y(x)\right) \varphi(x, y) .
$$

Note that when $Y$ and $\mathcal{V}$ are constant families, we have $\left(\Sigma_{\mathfrak{U}} x \in X\right) \mathcal{V}=\mathcal{U} \otimes \mathcal{V}$, so that indexed sum really does generalise tensor product. Like before, we can obtain an explicit formula for indexed sum by instantiating at the characteristic functions of predicates, and like before, we have a formula relating indexed sums with ultraproducts; this is now the general case of [17, Theorem 1.10].

Proposition 9. Given $\mathcal{U} \in \beta X$ and $\mathcal{V} \in(\Pi x \in X) \beta(Y(x))$ and an $X . Y$-indexed family of sets $Z$, currying of functions induces an isomorphism of ultraproducts

$$
\left(\Pi_{\mathcal{U} . \mathcal{V}}(x, y) \in X . Y\right) Z(x, y) \cong\left(\Pi_{\mathcal{U}} x \in X\right)\left(\Pi_{\mathcal{V}(x)} y \in Y(x)\right) Z(x, y) .
$$

\section{The MAIN THEOREM}

In this section, we prove our main theorem. This makes essential use of Börger's characterisation [9] of the ultrafilter endofunctor, so we begin by recalling this.

3.1. Börger's theorem. If $u: \mathcal{P} X \rightarrow 2$ is a Boolean algebra homomorphism and $f: X \rightarrow Y$, then $u \circ\left(f^{-1}\right): \mathcal{P} Y \rightarrow \mathcal{P} X \rightarrow 2$ is again a homomorphism, called the pushforward of $u$ along $f$. Identifying $u$ with the corresponding $\mathcal{U} \subseteq \mathcal{P} X$, its pushforward along $f$ is given by:

$$
f_{!}(\mathcal{U})=\left\{V \subseteq Y: f^{-1}(V) \in \mathcal{U}\right\} .
$$


Definition 10. The ultrafilter endofunctor $\beta$ : Set $\rightarrow$ Set has action on objects $X \mapsto \beta X$ and action on morphisms $\beta f: \beta X \rightarrow \beta Y$ given by $\mathcal{U} \mapsto f_{!}(\mathcal{U})$.

In [9] Börger characterises $\beta$ as terminal in the category FC(Set, Set) of finitecoproduct-preserving endofunctors of Set. In reproducing the proof, and subsequently, we will use the following lemma, whose proof is either an easy exercise for the reader, or a consequence of the more general Lemma 19 below. In the statement, we call a natural transformation $\alpha: F \Rightarrow G: \mathcal{C} \rightarrow \mathcal{D}$ monocartesian if the naturality square of $\alpha$ at any monomorphism $f: X \mapsto Y$ is a pullback.

Lemma 11. Let $G$ : Set $\rightarrow$ Set preserve finite coproducts and let $\alpha: F \Rightarrow G$.

(i) $G$ preserves monomorphisms and pullbacks along monomorphisms;

(ii) $F$ preserves finite coproducts if and only if $\alpha$ is monocartesian.

Theorem 12. [9, Theorem 2.1] $\beta$ is terminal in $\mathrm{FC}($ Set, Set).

Proof. For an injection $f: X \rightarrow Y$, the map $\beta f: \beta X \rightarrow \beta Y$ is also injective with

$$
\operatorname{im} \beta f=\{\mathcal{V} \in \beta Y: f(X) \in \mathcal{V}\} .
$$

Indeed, since $f$ is injective, $f^{-1}: \mathcal{P} Y \rightarrow \mathcal{P} X$ is surjective and so $\beta f=(-) \circ\left(f^{-1}\right)$ is injective. As for its image: each $\mathcal{U} \in \beta X$ contains $X=f^{-1}(f(X))$, so by (3.1) each $f_{!}(\mathcal{U})$ contains $f(X)$. Conversely, if $\mathcal{V} \in \beta Y$ contains $f(X)$, then $\mathcal{U}=\{U \subseteq X: f(U) \in \mathcal{V}\}$ is an ultrafilter on $X$ with $f_{!}(\mathcal{U})=\mathcal{V}$.

We first use this to show $\beta \in \mathrm{FC}$ (Set, Set). Clearly $\beta(\emptyset)=\emptyset$; while if we have a coproduct $y_{1}: Y_{1} \rightarrow Y \leftarrow Y_{2}: y_{2}$, then the maps $\beta y_{1}: \beta Y_{1} \rightarrow \beta Y \leftarrow \beta Y_{2}: \beta y_{2}$ are each injective with as images the sets $A=\left\{\mathcal{U} \in \beta Y: \operatorname{im} y_{1} \in \mathcal{U}\right\}$ and $B=\left\{\mathcal{U} \in \beta Y: \operatorname{im} y_{2} \in \mathcal{U}\right\}$. Since $\operatorname{im} y_{1}$ and $\operatorname{im} y_{2}$ partition $Y$, each $\mathcal{U} \in \beta Y$ lies in exactly one of $A$ or $B$ whence $\left(\beta y_{1}, \beta y_{2}\right)$ is again a coproduct cone.

We now show $\beta$ is terminal in $\mathrm{FC}($ Set, Set). Given $T \in \mathrm{FC}($ Set, Set $)$ and $x \in T X$, define the type of $x$ as the ultrafilter on $X$ given by:

$$
\tau_{X}(x)=\{U \subseteq X: x \text { is in the image of the monic } T U \longmapsto T X\} .
$$

Here, $T U \longmapsto T X$ is the $T$-image of the inclusion $U \subseteq X$, and so monic by Lemma 11. $\tau_{X}(x)$ satisfies axiom (i) for an ultrafilter since $T$ preserves pullbacks of monics, and satisfies (ii) ${ }^{\prime}$ as $T U \longmapsto T X \longleftarrow T(X \backslash U)$ is the $T$-image of a coproduct diagram and so itself a coproduct.

So we have functions $\tau_{X}: T X \rightarrow \beta X$. To verify their naturality in $X$ we must show for any $x \in T X$ and $f: X \rightarrow Y$ that $\tau_{Y}(T f(x))=f_{!}\left(\tau_{X}(x)\right)$. So for any $V \subseteq Y$, we must show $T f(x) \in T Y$ is in the image of $T V \longmapsto T Y$ if and only if $x \in T X$ is in the image of $T\left(f^{-1}(V)\right) \longmapsto T X$; which is so because $T$ preserves the pullback of $V \longmapsto Y$ along $f: X \rightarrow Y$ by Lemma 11. So we have $\tau: T \Rightarrow \beta$.

Finally, we check uniqueness of $\tau$. Any $\sigma: T \Rightarrow \beta$ is monocartesian by Lemma 11; and so for each $m: U \subseteq X$ the following square is a pullback:

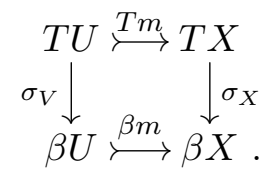


Thus, $x \in T X$ factors through $T m$ if and only if $\sigma_{X}(x)$ factors through $\beta m$ which by (3.2) happens just when $U \in \sigma_{X}(x)$. So $\sigma_{X}(x)=\tau_{X}(x)$ as desired.

3.2. The main theorem. We now exploit Börger's theorem to prove our main Theorem 13. In doing so, we make use of the well-known generalisation of (2.1) stating that any slice of a presheaf category is equivalent to a presheaf category.

Indeed, given $X \in[\mathcal{A}$, Set $]$, the category of elements el $X$ has as objects, pairs $(A \in \mathcal{A}, x \in X A)$ and as morphisms $(A, x) \rightarrow\left(A^{\prime}, x^{\prime}\right)$, maps $f: A \rightarrow A^{\prime}$ in $\mathcal{A}$ such that $x^{\prime}=X f(x)$. The equivalence in question is now

$$
[\mathrm{el} X, \text { Set }] \simeq[\mathcal{A}, \text { Set }] / X
$$

and is constructed by applying (2.1) componentwise as follows. Going from left to right, $Y:$ el $X \rightarrow$ Set is sent to $\pi: \int Y \rightarrow X$ whose $A$-component is given by the first projection map $(\Sigma x \in X A) Y(A, x) \rightarrow X A$, and where the action of $\int Y$ on maps is induced from those of $X$ and $Y$. Going from right to left, $p: E \rightarrow X$ in $[\mathcal{A}, \operatorname{Set}] / X$ is sent to $\tilde{E}$ in $[$ el $X$, Set $]$ with $\tilde{E}(A, x)=p_{A}^{-1}(x) \subseteq E A$ and action on maps inherited from $E$. For a detailed proof of the equivalence, see for example [25, Proposition A1.1.7].

Theorem 13. The category $\mathrm{FC}(\mathcal{S e t}$, Set) of finite coproduct-preserving endofunc-

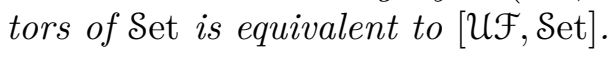

Proof. Note that $T \in$ [Set, Set] preserves finite coproducts if and only if it admits a monocartesian transformation to $\beta$, which is then necessarily unique. The "if" direction of this claim follows from Lemma 11; whereupon the "only if" direction and the unicity follow from Theorem 12. So we have an isomorphism of categories

$$
\mathrm{FC}(\text { Set, Set }) \cong[\text { Set, Set }] / \mathrm{mc} \beta
$$

where to the right we have the full, replete, subcategory [Set, Set] $/ \mathrm{mc} \beta$ of $[$ Set, Set $] / \beta$ on the monocartesian arrows.

Now, the full slice category [Set, Set] $/ \beta$ is equivalent to $[\mathrm{el} \beta$, Set]. Here, objects of el $\beta$ are pairs $(X \in \mathcal{S}$ et, $\mathcal{U} \in \beta X)$, while maps $(X, \mathcal{U}) \rightarrow(Y, \mathcal{V})$ are functions $f: X \rightarrow Y$ such that $f_{!}(\mathcal{U})=\mathcal{V}$. Comparing (2.4) with (3.1), these are exactly the continuous maps, so that el $\beta \cong \mathcal{U} \mathcal{E}$ and (3.3) becomes an equivalence:

$$
[\text { Set, Set }] / \beta \simeq[\mathcal{U E}, \text { Set }] \text {. }
$$

An object $\tau: T \Rightarrow \beta$ to the left of this equivalence lies in the full replete subcategory [Set, Set] /mc $\beta$ just when for each monic $f: X \longmapsto Y$ and $\mathcal{U} \in \beta X$, the map on fibres $\tau_{X}^{-1}(\mathcal{U}) \rightarrow \tau_{Y}^{-1}\left(f_{!}(\mathcal{U})\right)$ is an isomorphism. This is equally the condition that the corresponding $\bar{\tau} \in[\mathcal{U} \mathcal{E}$, Set] to the right lies in the full, replete subcategory of functors which send the class $\mathcal{M}$ of continuous injective functions to isomorphisms. By Proposition 5, this subcategory is isomorphic to $[\mathcal{U F}$, Set] via restriction along $\iota: \mathcal{U E} \rightarrow \mathcal{U F}$. So (3.5) restricts to an equivalence $[$ Set, Set] $/ \mathrm{mc} \beta \simeq[\mathcal{U F}$, Set], and combining this with (3.4) yields the desired equivalence $\mathrm{FC}($ Set, Set $) \simeq[\mathcal{U F}$, Set $]$.

Chasing through the above equivalences, we see that for each $A \in \mathrm{FC}$ (Set, Set), the corresponding $\tilde{A}: U \mathcal{F} \rightarrow$ Set is defined on objects by

$$
\tilde{A}(X, \mathcal{U})=\left\{x \in A X: \tau_{X}(x)=\mathcal{U}\right\} \cong \bigcap_{U \in \mathcal{U}} \operatorname{im} A U \subseteq A X .
$$


For its definition on morphisms, let the map $(X, \mathcal{U}) \rightarrow(Y, \mathcal{V})$ of $\mathcal{U F}$ be represented by the partial continuous $f: X \rightarrow Y$ defined on $U \in \mathcal{U}$. Then the induced function $\tilde{A}(X, \mathcal{U}) \rightarrow \tilde{A}(Y, \mathcal{V})$ is defined by $x \mapsto A f\left(x^{\prime}\right)$, where $x^{\prime} \in A U$ is the lifting of $x$ through $A U \longmapsto A X$ guaranteed by the fact that $U \in \tau_{X}(x)$.

In the other direction, for any $B: \mathcal{U F} \rightarrow$ Set, the corresponding finitecoproduct-preserving $\int B$ : Set $\rightarrow$ Set is defined by

$$
\left(\int B\right) Y=\sum_{\mathcal{V} \in \beta Y} B(Y, \mathcal{V}) \quad \text { and } \quad\left(\int B\right) f:(\mathcal{V}, a) \mapsto\left(f_{!}(\mathcal{V}), A f(a)\right) .
$$

3.3. Relation to ultrapowers and tensor products. We now show that both ultrapowers and the tensor product of ultrafilters arise naturally from the preceding equivalence. We begin with ultrapowers.

Corollary 14. Under the equivalence of Theorem 13, the representable functor $\mathcal{U F}((X, \mathcal{U}),-): \mathcal{U F} \rightarrow$ Set corresponds to the ultrapower functor $(-)^{\mathfrak{U}}:$ Set $\rightarrow$ Set.

Proof. Taking $B$ to be $y_{(X, \mathcal{U})}=\mathcal{U F}((X, \mathcal{U}),-)$ in (3.7), we have that

$$
\left(\int y_{(X, \mathcal{U})}\right)(X)=\sum_{\mathcal{v} \in \beta Y} \mathfrak{U F}((X, \mathcal{U}),(Y, \mathcal{v})) .
$$

An element of this set is a pair $\mathcal{V} \in \beta Y$ together with an $=u$-equivalence class of continuous partial functions $f:(X, \mathcal{U}) \rightarrow(Y, \mathcal{V})$ defined on a set in $\mathcal{U}$. The continuity condition (2.4) forces $\mathcal{V}=f_{!}(\mathcal{U})$ and so this is equally a $=\mathfrak{u}$-equivalence class of partial functions $f: X \rightarrow Y$ defined on a set in $\mathcal{U}$; thus an element of the ultrapower $Y^{\mathfrak{U}}$. This proves that $\int y_{(X, \mathcal{U})} \cong(-)^{\mathfrak{U}}$ as desired.

As remarked in the introduction, we can use this result to recover the category $\mathcal{U F}$ from $\mathrm{FC}(\mathcal{S e t}$, Set). Recall that an object $X$ of a category $\mathcal{E}$ is small-projective if the hom-functor $\mathcal{E}(X,-): \mathcal{E} \rightarrow$ Set preserves all small colimits.

Corollary 15. The category UF ${ }^{\circ}$ is equivalent to the full subcategory of $\mathrm{FC}(\mathcal{S e t}$, Set) on the small-projectives.

Proof. For any locally small $\mathcal{A}$, the small-projectives in $[\mathcal{A}, \mathcal{S}$ et $]$ are precisely the retracts of representable functors; see, for example, [8, Lemma 6.5.10]. So if representables are closed under retracts, then $\mathcal{A}^{\text {op }}$ is equivalent to the full subcategory of $[\mathcal{A}$, Set $]$ on the small-projectives.

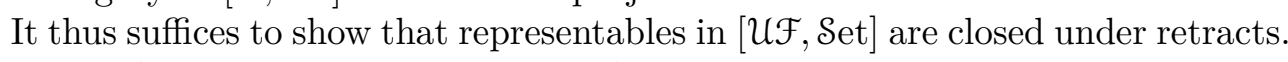

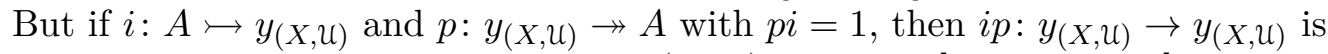
the image under $y$ of an idempotent on $(X, \mathcal{U})$. Since by [5, Theorem 5$]$, the only idempotents (indeed, the only endomorphisms) in $\mathcal{U F}$ are the identities, we thus have $i p=1$ as well as $p i=1$, so that $A \cong y_{(X, \mathcal{U})}$ is again representable.

We now turn to the tensor product of ultrafilters. The category FC(Set, Set) has a monoidal structure given by composition, and transporting this across the equivalence of Theorem 13 yields a monoidal structure $(I, \otimes)$ on [UF, Set].

Proposition 16. The representables in [UF, Set] are closed under the monoidal structure, and the induced monoidal structure on $\mathrm{UF}$ is that given by tensor product of ultrafilters. 
Proof. The identity functor Set $\rightarrow$ Set corresponds to the functor UF $\rightarrow$ Set represented by the unique ultrafilter on a one-element set, which is the unit for the monoidal structure on $\mathcal{U F}$. On the other hand, if $A, B \in[\mathcal{U F}$, Set] are represented by $(X, \mathcal{U})$ and $(Y, \mathcal{V})$ respectively, then by Theorem 13 we have $\int A \cong(-)^{\mathfrak{U}}$ and $\int B \cong(-)^{\mathcal{V}}$, and so $\int A \circ \int B \cong\left((-)^{\mathcal{V}}\right)^{\mathcal{U}} \cong(-)^{\mathcal{U} \otimes \mathcal{V}}$ by Proposition 7 . It follows that $A \otimes B$ is represented by $(X \times Y, \mathcal{U} \otimes \mathcal{V})=(X, \mathcal{U}) \otimes(Y, \mathcal{V})$ in $\mathcal{U F}$.

The monoidal structure on [UFF, Set] is easy to write down explicitly. As already noted, the unit $I$ is the functor representable at the unique ultrafilter on the one-element set, while the binary tensor can be given as $A \otimes B=\int A \circ B$, where $\int A$ is defined as in (3.7). This yields the formulae:

$$
\begin{aligned}
I(X, \mathcal{U}) & = \begin{cases}1 & \text { if } \mathcal{U} \text { is principal } \\
0 & \text { otherwise. }\end{cases} \\
(A \otimes B)(X, \mathcal{U}) & =\sum_{\mathcal{V} \in \beta(B(X, \mathcal{U}))} A(B(X, \mathcal{U}), \mathcal{V}) .
\end{aligned}
$$

We have an alternative description of the binary tensor product by exploiting the fact that, since the composition product on $\mathrm{FC}(\mathcal{S e t}$, Set) preserves colimits in its first variable, so too does the tensor product $\otimes$ on [UF , Set]:

$$
\begin{aligned}
(A \otimes B)(X, \mathcal{U}) & \cong\left(\left(\int^{(Y, \mathcal{V}) \in \mathcal{U F}} A(Y, \mathcal{V}) \times y_{(Y, \mathcal{V})}\right) \otimes B\right)(X, \mathcal{U}) \\
& \cong \int^{(Y, \mathcal{V}) \in \mathcal{U F}} A(Y, \mathcal{V}) \times\left(y_{(Y, \mathcal{V})} \otimes B\right)(X, \mathcal{U}) \\
& \cong \int^{(Y, \mathcal{V}) \in \mathcal{U F}} A(Y, \mathcal{V}) \times B(X, \mathcal{U})^{\mathcal{V}}
\end{aligned}
$$

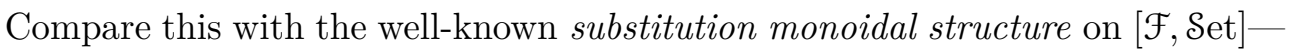
for $\mathcal{F}$ the category of functions between finite cardinals - defined by:

$$
(A \otimes B)(m)=\int^{n \in \mathcal{F}} A n \times B m^{n} .
$$

Remark 17. Given a monoidal category $\mathcal{V}$, one may consider categories enriched over $\mathcal{V}$ in the sense of [31]. A $\mathcal{V}$-enriched category $\mathcal{C}$ involves a set of objects $A, B, C, \ldots$ as usual, but instead of hom-sets of morphisms, one has hom-objects $\mathcal{C}(A, B)$ in $\mathcal{V}$. In the enriched context, one has a new kind of colimit available, namely the copower $V \cdot A$ of an object $A \in \mathcal{C}$ by an object $V \in \mathcal{V}$, characterised by natural isomorphisms $\mathrm{C}(V \cdot A, B) \cong[V, \mathcal{C}(A, B)]$ in $\mathcal{V}$.

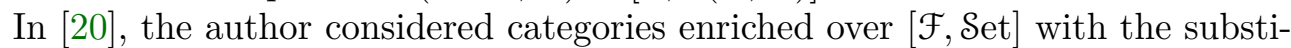
tution monoidal structure, and showed that such [F, Set]-categories admitting copowers by representables correspond to ordinary categories $\mathcal{C}$ admitting finite powers $(-)^{n}: \mathcal{C} \rightarrow \mathcal{C}$. The analogy between (3.8) and (3.9) suggests that some-

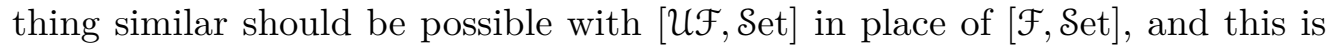

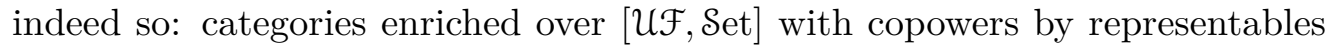
correspond to ordinary categories $\mathcal{C}$ equipped with abstract ultrapower functors $(-)^{\mathfrak{U}}: \mathcal{C} \rightarrow \mathcal{C}$. The details of this will be left for future work, but we discuss an extension to categories endowed with abstract ultraproduct functors in Remark 32 below. 


\section{First generalisation}

In this section, we give our first generalisation of Theorem 13. This will show that, for any extensive category $\mathcal{C}$, there is an equivalence $\mathrm{FC}(\mathcal{C}, \mathcal{S}$ et $) \simeq$ $\left[\mathcal{U} \mathcal{F}_{\mathcal{C}}, \mathcal{S}\right.$ et $]$ where $\mathcal{U} \mathcal{F}_{\mathcal{C}}$ is a suitably defined category of ultrafilters on $\mathcal{C}$-objects. We then describe how this result captures the notion of ultraproduct, and how it reconstructs the categorical treatment in [38] of types in model theory.

4.1. Generalising the domain category. We begin by recalling from [12] that a category $\mathcal{C}$ with finite coproducts is extensive if for every $A, B \in \mathcal{C}$, the functor $+: \mathcal{C} / A \times \mathcal{C} / B \rightarrow \mathcal{C} /(A+B)$ is an equivalence of categories. Equally, by [12, Proposition 2.2], $\mathcal{C}$ is extensive just when it has pullbacks along coproduct coprojections, and, for every diagram

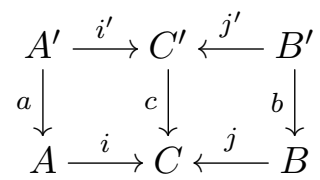

in which the bottom row is a coproduct diagram, the top row is a coproduct diagram if and only if the two squares are pullbacks.

Note the "if" direction says that binary coproducts in $\mathcal{C}$ are pullback-stable. In fact, a category with finite coproducts and pullbacks along their coprojections is extensive just when binary coproducts are pullback-stable and disjoint; see [12, Proposition 2.14]. Disjointness means that coproduct coprojections are monic, and the pullback of the two coprojections of a binary coproduct is initial.

This characterisation implies that any topos is extensive; see [25, Proposition A2.3.4 \& Corollary A2.4.4]. In particular, Set is extensive, as is any presheaf category. Other examples of extensive categories include the categories of topological spaces, of small categories and of affine schemes.

Let us write $\operatorname{Sum}_{\mathcal{C}}(X)$ for the poset of coproduct summands of $X \in \mathcal{C}$ : that is, the poset of isomorphism-classes of coproduct coprojections with codomain $X$.

Proposition 18. If $\mathcal{C}$ is extensive, then for each $X \in \mathcal{C}$ the poset $\operatorname{Sum}_{\mathcal{C}}(X)$ is a Boolean algebra, and for each $f: X \rightarrow Y$, pullback along $f$ defines a Boolean algebra homomorphism $f^{-1}: \operatorname{Sum}_{\mathfrak{C}}(Y) \rightarrow \operatorname{Sum}_{\mathcal{C}}(X)$.

Proof. Since binary coproducts in $\mathcal{C}$ are stable under pullback, so are coproduct coprojections; since they are also composition-closed, each $\operatorname{Sum}_{\mathcal{C}}(X)$ has finite meets, and $f^{-1}: \operatorname{Sum}_{\mathcal{C}}(Y) \rightarrow \operatorname{Sum}_{\mathcal{C}}(X)$ is well-defined and finite-meet-preserving. Now any $Y_{1} \longmapsto Y$ in $\operatorname{Sum}_{\mathcal{C}}(X)$ is part of a coproduct $Y_{1} \longmapsto Y \longleftrightarrow Y_{2}$. Of course $Y_{1} \cup Y_{2}=\top_{Y}$ in $\operatorname{Sum}_{\mathcal{C}}(Y)$, and $Y_{1} \cap Y_{2}=\perp_{Y}$ by disjointness; so $\operatorname{Sum}_{\mathcal{C}}(X)$ has complements and so is a Boolean algebra. Further, $f^{-1}: \operatorname{Sum}_{\mathcal{C}}(Y) \rightarrow \operatorname{Sum}_{\mathfrak{C}}(X)$ preserves these complements as binary coproducts are pullback-stable.

A case worth noting is that where $\mathcal{C}$ is Boolean extensive, meaning that every monic in $\mathcal{C}$ is a coproduct coprojection; in this situation $\operatorname{Sum}_{\mathfrak{C}}(X)$ coincides with the full subobject lattice $\operatorname{Sub}_{\mathcal{C}}(X)$, so that all subobject lattices in $\mathcal{C}$ are Boolean algebras - whence the nomenclature. In particular, the category of sets is Boolean extensive, so that the following result is a generalisation of Lemma 11 above. In 
the last part of the statement, a natural transformation $\alpha$ is called sum-cartesian if its naturality square at every coproduct coprojection is a pullback.

Lemma 19. Let $\mathcal{C}$ and $\mathcal{D}$ be extensive, let $G: \mathcal{C} \rightarrow \mathcal{D}$ be finite-coproduct-preserving and let $\alpha: F \Rightarrow G: \mathcal{C} \rightarrow \mathcal{D}$.

(i) $G$ preserves both coproduct coprojections and pullbacks along such;

(ii) $F$ preserves finite coproducts just when $\alpha: F \Rightarrow G$ is sum-cartesian.

Proof. The first part of (i) is clear. For the second, any pullback along a coproduct coprojection in $\mathcal{C}$ is the left square of a diagram like (4.1) in which both rows are coproducts. Applying $F$, both rows remain coproducts and so by extensivity of $\mathcal{D}$, both squares remain pullbacks. As for (ii), given a coproduct diagram $i: A \rightarrow C \leftarrow B: j$ in $\mathcal{C}$, we consider the diagram

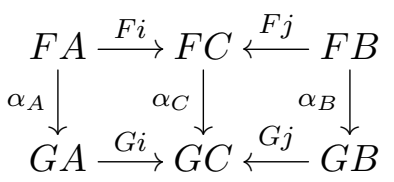

in $\mathcal{D}$. The bottom row is a coproduct since $G$ preserves such; so, by extensivity of $\mathcal{D}$, the top row is a coproduct (i.e., $F$ preserves finite coproducts) just when both squares are pullbacks (i.e., $\alpha$ is sum-cartesian).

If $\mathcal{C}$ is extensive, then we define an ultrafilter on $X \in \mathcal{C}$ to be a Boolean algebra homomorphism $\operatorname{Sum}_{\mathcal{C}}(X) \rightarrow 2$; equivalently, a subset $\mathcal{U} \subseteq \operatorname{Sum}_{\mathfrak{C}}(X)$ satisfying the analogue of conditions (i) and (ii) or (i) and (ii)' of Definition 2. Like before, we write $\beta X$ for the set of ultrafilters on $X \in \mathcal{C}$. Since each $f^{-1}: \operatorname{Sum}_{\mathcal{C}}(Y) \rightarrow \operatorname{Sum}_{\mathcal{C}}(X)$ is a Boolean algebra homomorphism, precomposition with $f^{-1}$ yields a function $\beta f: \beta X \rightarrow \beta Y$; in this way, we define an ultrafilter functor $\beta: \mathcal{C} \rightarrow$ Set.

Proposition 20. $\beta$ : $\mathcal{C} \rightarrow$ Set is terminal in $\mathrm{FC}(\mathcal{C}$, Set $)$.

Proof. The proof in Theorem 12 adapts without difficulty to show that $\beta: \mathcal{C} \rightarrow$ Set preserves finite coproducts. To show terminality in $\mathrm{FC}(\mathcal{C}$, Set $)$, we suppose given $T \in \mathrm{FC}(\mathcal{C}, \mathcal{S e t})$. For any $X \in \mathcal{C}$ and $x \in T X$, we again define the type of $x$ to be:

$$
\tau_{X}(x)=\left\{U \stackrel{m}{\longmapsto} X \in \operatorname{Sum}_{\mathcal{C}}(X): x \text { factors through } F U \stackrel{F m}{\longmapsto} F X\right\} .
$$

The same argument as before, but now exploiting Lemma 19 in place of Lemma 11, shows that this definition gives the values of a well-defined natural transformation $\tau: T \Rightarrow \beta: \mathcal{C} \rightarrow \operatorname{Set}$, and that this $\tau$ is unique.

Like before, given objects $X, Y \in \mathcal{C}$ endowed with ultrafilters $\mathcal{U}$ and $\mathcal{V}$, we call $f: X \rightarrow Y$ continuous if for all $V \in \operatorname{Sum}_{\mathcal{C}}(Y)$ we have $V \in \mathcal{V} \Leftrightarrow f^{-1}(V) \in \mathcal{U}$. More generally, a map $f: U \rightarrow Y$ defined on the domain of some $U \longmapsto X$ in $\mathcal{U}$ is continuous if $V \longmapsto Y \in \mathcal{V}$ just when $f^{-1}(V) \longmapsto U \longmapsto X \in \mathcal{U}$. Two partial maps defined on $U$ and $U^{\prime}$ are $=u$-equivalent if their restrictions to some $W \subseteq U \cap U^{\prime}$ in $\mathcal{U}$ coincide. 
Definition 21. The category $\mathcal{U} \mathcal{E}_{\mathcal{e}}$ has pairs $(X \in \mathcal{C}, \mathcal{U} \in \beta X)$ as objects, and as morphisms $(X, \mathcal{U}) \rightarrow(Y, \mathcal{V})$ the continuous maps $X \rightarrow Y$. The category $\mathcal{U} \mathcal{F}_{\mathcal{C}}$ has the same objects, and as morphisms $(X, \mathcal{U}) \rightarrow(Y, \mathcal{V})$ the $=\mathfrak{u}$-equivalence classes of partial continuous maps $X \rightarrow Y$ defined on the domain of some $U \mapsto X$ in $\mathcal{U}$.

Writing $\mathcal{M}_{\Sigma}$ for the maps in $\mathcal{U E}_{\mathcal{E}}$ whose underlying map in $\mathcal{C}$ is a coproduct coprojection, we have as in Proposition 5, that $\mathcal{U F}_{\mathcal{C}} \cong \mathcal{U}_{\mathcal{E}}\left[\mathcal{M}_{\Sigma}^{-1}\right]$. Now transcribing the proof of Theorem 13 and Corollary 14, but exploiting Proposition 20 and Lemma 19 in place of Theorem 12 and Lemma 11, gives the following.

Theorem 22. Let $\mathcal{C}$ be extensive. The category $\mathrm{FC}(\mathcal{C}$, Set $)$ of finite coproduct-

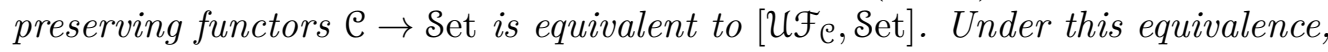
the representable presheaf at $(X, \mathcal{U}) \in \mathcal{U} \mathcal{F}_{\mathcal{C}}$ corresponds to the "ultrahom functor"

$$
\mathcal{C}(X,-) \mathfrak{u}=\operatorname{colim}_{U \in \mathfrak{U}} \mathcal{C}(U,-): \mathcal{C} \rightarrow \text { Set } .
$$

The formulae for the two directions of the equivalence $\mathrm{FC}(\mathcal{C}, \mathcal{S}$ et $) \simeq\left[\mathcal{U} \mathcal{F}_{\mathcal{C}}\right.$, Set $]$ are once again given by (3.6) and (3.7).

Example 23. The category Stone of Stone spaces is extensive and $\operatorname{Sum}_{\mathcal{S} t o n e}(X)$ is the Boolean algebra of clopen sets of $X$. It follows by Stone duality that ultrafilters on $X \in$ Stone correspond exactly to points of $X$, so that the category $\mathcal{U} \mathcal{F}_{\text {Stone }}$ has pointed Stone spaces $(X, x)$ as objects, and as maps $f:(X, x) \rightarrow(Y, y)$, germs at $x$ of point-preserving continuous functions $X \rightarrow Y$. Under the equivalence $\left[\mathcal{U F}_{\text {Stone }}, \mathcal{S e t}\right] \simeq \mathrm{FC}($ Stone, Set $)$, the representable at $(X, x)$ corresponds to the functor which sends a Stone space $Y$ to the stalk at $x$ of the sheaf of continuous functions $X \rightarrow Y$.

4.2. Relation to ultraproducts. We now explain how Theorem 22 allows us to reconstruct the notion of ultraproduct. Taking $\mathcal{C}=\operatorname{Set}^{X}$ therein yields the equivalence $\left[\mathcal{U} \mathcal{F}_{\operatorname{Set}^{X}}\right.$, Set $] \simeq \mathrm{FC}\left(\mathcal{S e t}^{X}\right.$, Set $)$, and we will obtain the ultraproduct functors as correlates to the right of suitable representable functors to the left.

Note first that, via (2.1), we have for any $A \in \operatorname{Set}^{X}$ that

$$
\operatorname{Sum}_{\mathcal{S e t}^{X}}(A) \cong \operatorname{Sum}_{\mathcal{S e t} / X}\left(\pi_{A}: X . A \rightarrow X\right) \cong \mathcal{P}(X . A),
$$

so that ultrafilters on $A \in \operatorname{Set}^{X}$ can be identified with ultrafilters on $X$.A in Set. Under this identification, the ultrafilter $\mathcal{U}$ on $X$.A corresponds to the ultrafilter $\tilde{\mathcal{U}}=\{\tilde{U}: U \in \mathcal{U}\}$ on $A$ composed of the subobjects $\tilde{U} \longmapsto A$ obtained by passing the subobjects $U \longmapsto X . A \rightarrow X$ of $\pi_{A}: X . A \rightarrow X$ across the equivalence (2.1).

Proposition 24. Under the equivalence $\left[\mathcal{U F}_{\mathcal{S e t}^{X}}\right.$, Set $] \simeq \mathrm{FC}\left(\operatorname{Set}^{X}\right.$, Set $)$, the representable functor at $\left(A \in \operatorname{Set}^{X}, \mathfrak{U} \in \beta(X . A)\right)$ corresponds to the composite

$$
\operatorname{Set}^{X} \stackrel{\operatorname{Set}^{\pi_{A}}}{\longrightarrow} \operatorname{Set}^{X . A} \stackrel{\Pi_{\mathcal{U}}}{\longrightarrow} \operatorname{Set} .
$$

In particular, the representable at $(1, \mathcal{U})$ corresponds to $\Pi_{\mathfrak{U}}: \operatorname{Set}^{X} \rightarrow \operatorname{Set}$. 
Proof. From Theorem 22 and the above remarks, we know that $y_{(A, u)}$ corresponds to the ultrahom functor $\operatorname{Set}^{X}(A,-) \tilde{u}$. We now calculate that:

$$
\begin{aligned}
\operatorname{Set}^{X}(A, Y)_{\tilde{\mathcal{U}}} & =\operatorname{colim}_{\tilde{U} \in \tilde{\mathcal{U}}} \operatorname{Set}^{X}(\tilde{U}, Y) \\
& \cong \operatorname{colim}_{U \in \mathcal{U}} \operatorname{Set} / X\left(U \longmapsto X . A \stackrel{\pi_{A}}{\longrightarrow} X, X . Y \stackrel{\pi_{Y}}{\longrightarrow} X\right) \\
& \cong \operatorname{colim}_{U \in \mathcal{U}} \operatorname{Set} / X . A\left(U \longmapsto X . A, \pi_{A}^{*}(X . Y) \stackrel{\pi_{A}^{*} \pi_{Y}}{\longrightarrow} X . A\right) \\
& \cong \operatorname{colim}_{U \in \mathcal{U}}(\Pi x \in U) Y\left(\pi_{A}(x)\right)=\left(\Pi_{\mathcal{U}} x \in X . A\right) Y\left(\pi_{A}(x)\right),
\end{aligned}
$$

so that $y_{(A, \mathcal{U})}$ corresponds to the composite (4.2) as desired.

4.3. Relation to model theory. Finally in this section, we relate Theorem 22 to types in model theory. Recall that, if $\mathbb{T}$ is a (classical, single-sorted) first-order theory, and we write $\mathbb{T}\left[x_{1}, \ldots, x_{n}\right]$ for the theory obtained by adjoining new constants $x_{1}, \ldots, x_{n}$ to $\mathbb{T}$, then a complete $n$-type of $\mathbb{T}$ is a complete theory extending $\mathbb{T}\left[x_{1}, \ldots, x_{n}\right]$; equally, it is an ultrafilter on the Lindenbaum-Tarski algebra $B_{\mathbb{T}\left[x_{1}, \ldots, x_{n}\right]}$ of sentences in $\mathbb{T}\left[x_{1}, \ldots, x_{n}\right]$ identified up to provable equivalence.

Each $n$-tuple of elements $\vec{a}=a_{1}, \ldots, a_{n}$ in a $\mathbb{T}$-model $A$ yields a complete $n$-type $\tau(\vec{a})$, namely the set of all sentences $\varphi\left(x_{1}, \ldots, x_{n}\right)$ of $\mathbb{T}\left[x_{1}, \ldots, x_{n}\right]$ such that $A \vDash \varphi\left(a_{1}, \ldots, a_{n}\right)$; we say that the $n$-tuple $\vec{a}$ realises the type $\tau(\vec{a})$. Many important questions in model theory revolve around the existence, or otherwise, of tuples of elements which realise a given type. As we now show, we can obtain the realisation relation between $n$-types of $\mathbb{T}$ and $n$-tuples in a $\mathbb{T}$-model by applying Theorem 22 with $\mathcal{C}$ taken to be the classifying Boolean pretopos of $\mathbb{T}$.

The classifying Boolean pretopos of a first-order theory has a characterising property analogous to that of the Lindenbaum-Tarski algebra $B_{\mathbb{P}}$ of a (classical) propositional theory $\mathbb{P}$. Indeed, there is a standard notion of model of a propositional theory $\mathbb{P}$ in a Boolean algebra $C$, and the Lindenbaum-Tarski algebra $B_{\mathbb{P}}$ of all $\mathbb{P}$-sentences modulo $\mathbb{P}$-provable equality, is the universal Boolean algebra which models $\mathbb{P}$. By this, we mean that models of $\mathbb{P}$ in a Boolean algebra $C$ are in bijection with Boolean homomorphisms $B_{\mathbb{P}} \rightarrow C$.

The classifying Boolean pretopos of a first-order theory $\mathbb{T}$ has a similar property: it is the universal Boolean pretopos which models $\mathbb{T}$. We now make this precise. First, a pretopos is a category which is finitely complete, extensive and also Barrexact [1], meaning that it has well-behaved quotients of equivalence relations; while a pretopos is Boolean if it is so qua extensive category. If $\mathcal{C}$ and $\mathcal{D}$ are pretoposes, then a pretopos morphism $F: \mathcal{C} \rightarrow \mathcal{D}$ is a functor preserving finite limits, finite coproducts and regular epimorphisms; we write $\operatorname{Pretop}(\mathcal{C}, \mathcal{D})$ for the category of pretopos morphisms and all natural transformations.

There is a standard notion of model of a first-order theory $\mathbb{T}$ in a Boolean pretopos $\mathcal{C}$ - see, for example $[26, \S \mathrm{D} 1.2]$ - and these comprise the objects of a category $\mathbb{T}-\mathcal{M}_{\operatorname{lod}}(\mathcal{C})$ whose maps are elementary embeddings. If $\mathcal{C}$ and $\mathcal{D}$ are Boolean pretoposes, then any pretopos morphism $F: \mathcal{C} \rightarrow \mathcal{D}$ preserves $\mathbb{T}$-models and so induces a functor $F_{*}: \mathbb{T}-\mathcal{M}_{\operatorname{lod}}(\mathcal{C}) \rightarrow \mathbb{T}-\mathcal{M}_{\operatorname{lod}}(\mathcal{D})$.

Definition 25. A classifying Boolean pretopos for a first-order theory $\mathbb{T}$ is a Boolean pretopos $\mathcal{C} l(\mathbb{T})$ endowed with a $\mathbb{T}$-model $\mathbf{G} \in \mathbb{T}-\mathcal{M} \operatorname{lod}_{e}(\mathcal{C} l(\mathbb{T}))$ such that, 
for any Boolean pretopos $\mathcal{D}$, the following functor is an equivalence:

$$
\begin{aligned}
\operatorname{Pretop}(\mathcal{C l}(\mathbb{T}), \mathcal{D}) & \rightarrow \mathbb{T}-\mathcal{M l o d}_{e}(\mathcal{D}) \\
F & \mapsto F_{*}(\mathbf{G}) .
\end{aligned}
$$

To construct the classifying Boolean pretopos of a first-order theory $\mathbb{T}$, we first form its first-order syntactic category $\mathcal{C}_{\mathbb{T}}^{\text {fo }}$ whose objects are "formal $\mathbb{T}$-definable sets" $\{\vec{x}: \varphi(\vec{x})\}$ (i.e., first-order formulae-in-context) and whose maps are $\mathbb{T}$ provable equivalence classes of $\mathbb{T}$-provably functional relations from $\{\vec{x}: \varphi(\vec{x})\}$ to $\{\vec{y}: \psi(\vec{y})\}$; see, for example [26, $\S \mathrm{D} 1.4]$. The classifying Boolean pretopos $\mathrm{Cl}(\mathbb{T})$ is now obtained by freely adjoining finite coproducts and coequalisers of equivalence relations to $\mathcal{C}_{\mathbb{T}}^{\text {fo }}$ while preserving its existing finite unions and image factorisations - see [25, Proposition A1.4.5 \& Corollary A3.3.10] for the necessary constructions. Alternatively, we can obtain $\mathrm{Cl}(\mathbb{T})$ as the first-order syntactic category of $\mathbb{T}^{\mathrm{eq}}$, where $(-)^{\mathrm{eq}}$ is Shelah's elimination of imaginaries; this observation is due to Makkai and Reyes [42], and is explained in detail in [24].

We will not describe the generic $\mathbb{T}$-model $\mathbf{G}$ in $\mathcal{C l}(\mathbb{T})$ explicitly; however, part of its genericity is the fact that $\operatorname{Sum}_{\mathcal{C l}(\mathbb{T})}(G)$ is the Lindenbaum-Tarski algebra $B_{\mathbb{T}[x]}$. More generally $\mathrm{Sub}_{\mathcal{C l}(\mathbb{T})}\left(G^{n}\right)$ is the corresponding Lindenbaum-Tarski algebra $B_{\mathbb{T}\left[x_{1}, \ldots, x_{n}\right]}$. It follows that an ultrafilter on $G^{n} \in \mathcal{C l}(\mathbb{T})$ is exactly a complete $n$-type of $\mathbb{T}$. Now, since $\mathcal{D}=$ Set is a Boolean pretopos, we obtain from (4.3) and Theorem 22 a string of functors

$$
\mathbb{T}-\mathcal{M l o d}_{e}(\text { Set }) \stackrel{\simeq}{\longrightarrow} \operatorname{Pretop}(\mathcal{C l}(\mathbb{T}), \text { Set }) \stackrel{\subseteq}{\longrightarrow} \mathrm{FC}(\mathcal{C l}(\mathbb{T}), \text { Set }) \stackrel{\simeq}{\longrightarrow}\left[\mathcal{U} \mathcal{F}_{\mathrm{Cl} l(\mathbb{T})}, \text { Set }\right]
$$

assigning to each (ordinary) $\mathbb{T}$-model $\mathbf{M}$ both a functor $M: \mathcal{C l}(\mathbb{T}) \rightarrow$ Set and a functor $\tilde{M}: \mathcal{U F}(l(\mathbb{T}) \rightarrow$ Set. In this context, the passage from $M$ to $\tilde{M}$ was described by Makkai in [38], who also observed its model-theoretic import: it encodes the types realised by tuples of elements of the model $\mathbf{M}$.

Indeed, the pretopos morphsim $M: \mathcal{C l}(\mathbb{T}) \rightarrow$ Set corresponding to the model $\mathbf{M}$ sends $G$ to the underlying set $|\mathbf{M}|$ of the model, sends $G^{n}$ to $|\mathbf{M}|^{n}$ and sends $\varphi \in \operatorname{Sum}_{\mathcal{C l}(\mathbb{T})}\left(G^{n}\right)$ to the set $\left\{\vec{m} \in|\mathbf{M}|^{n}: \mathbf{M} \vDash \varphi(\vec{m})\right\}$. Thus, by (3.6), the value of the corresponding $\tilde{M} \in\left[\mathcal{U} \mathcal{F}_{\mathcal{l} l(\mathbb{T})}\right.$, Set $]$ at a complete $n$-type $\mathcal{U}$ is given by the set of $n$-tuples of elements of $\mathbf{M}$ which realise the type $\mathcal{U}$ :

$$
\tilde{M}\left(X^{n}, \mathcal{U}\right)=\left\{\vec{m} \in|\mathbf{M}|^{n}: \varphi \in \mathcal{U} \Longleftrightarrow \mathbf{M} \vDash \varphi(\vec{m})\right\} .
$$

\section{Second Generalisation}

In this section, we give our second generalisation of Theorem 13, which extends the first one to an equivalence $\mathrm{FC}(\mathcal{C}, \mathcal{E}) \simeq\left[\mathcal{U} \mathcal{F}_{\mathcal{C}}, \mathcal{E}\right]$, where $\mathcal{C}$ is extensive as before, and now $\mathcal{E}$ is any locally connected Grothendieck topos. We then use this result to reconstruct the indexed sum of ultrafilters, and in the process of doing so construct interesting and natural bicategories of ultramatrices and ultraspans. Finally, we describe how this relates to the ultracategories of [40].

5.1. Generalising the codomain category. A locally small category $\mathcal{E}$ is a Grothendieck topos if it is equivalent to the category of sheaves on a small site; see, for example, [36, Chapter III]. Equivalently, by Giraud's theorem (cf. [36, Appendix]), 
$\mathcal{E}$ is a Grothendieck topos just when it is finitely complete, Barr-exact and infinitary extensive with a small generating set.

In any Grothendieck topos $\mathcal{E}$, the functor $\Gamma=\mathcal{E}(1,-): \mathcal{E} \rightarrow$ Set has a left adjoint $\Delta$ : Set $\rightarrow \mathcal{E}$ which sends a set $X$ to the coproduct $\Sigma_{x \in X} 1$. We say that $\mathcal{E}$ is locally connected (or molecular [3]) if $\Delta$ has a further left adjoint $\pi_{0}: \mathcal{E} \rightarrow$ Set. For example, by [14, p.414, Ex. 7.6], the topos of sheaves on a space $X$ is locally connected just when $X$ is locally connected in the usual sense; in this case, $\pi_{0}: \mathcal{S h}(X) \rightarrow \mathcal{S}$ et sends a sheaf to the set of connected components of the corresponding étale space over $X$.

Theorem 26. Let $\mathcal{C}$ be extensive and $\mathcal{E}$ a locally connected Grothendieck topos. The category $\mathrm{FC}(\mathcal{C}, \mathcal{E})$ is equivalent to $\left[\mathcal{U F}_{\mathcal{C}}, \mathcal{E}\right]$ via an equivalence whose two directions are given by the formulae (3.6) and (3.7).

In proving this, we require a straightforward generalisation of the equivalence $[\mathrm{el} X, \operatorname{Set}] \simeq[\mathcal{A}, \operatorname{Set}] / X$ of (3.3) to an equivalence

$$
[\mathrm{el} X, \mathcal{E}] \simeq[\mathcal{A}, \mathcal{E}] / \Delta X
$$

for any Grothendieck topos $\mathcal{E}$. The generalisation makes use of the fact that $\mathcal{E}$ is infinitary extensive; this means that it has all small coproducts, and that for any set $X$, the coproduct functor $\Sigma: \prod_{x \in X}\left(\mathcal{E} / A_{x}\right) \rightarrow \mathcal{E} /\left(\Sigma_{i \in X} A_{x}\right)$ is an equivalence of categories. Taking each $A_{x}$ to be terminal and using $\mathcal{E} / 1 \cong \mathcal{E}$, we deduce that

$$
\Sigma: \mathcal{E}^{X} \rightarrow \mathcal{E} / \Delta X
$$

is an equivalence for any set $X$, with pseudoinverse given (necessarily) by pullback along the coproduct coprojections. By using this equivalence in place of (2.1), we may generalise (3.3) to the desired equivalence (5.1). Much as before, $Y \in$ [el $X, \mathcal{E}]$ is sent to $\pi: \int Y \rightarrow \Delta X$ whose component $\pi_{A}:\left(\int Y\right) A \rightarrow \Delta X A$ is the coproduct of the family of maps $(Y(A, x) \rightarrow 1)_{x \in X A}$; while conversely, $p: E \rightarrow X$ in $[\mathcal{A}, \mathcal{E}] / \Delta X$ is sent to $\tilde{E} \in[\mathrm{el} X, \mathcal{E}]$ wherein $\tilde{E}(A, x)$ is the pullback of $p_{A}: E A \rightarrow \Delta X A$ along $\Delta x: \Delta 1 \rightarrow \Delta X A$.

Proof of Theorem 26. Since $\pi_{0} \dashv \Delta \dashv \Gamma: \mathcal{E} \rightarrow$ Set, both $\pi_{0}$ and $\Delta$ preserve finite coproducts, so inducing an adjunction $\pi_{0} \circ(-) \dashv \Delta \circ(-): \mathrm{FC}(\mathcal{C}$, Set $) \rightarrow \mathrm{FC}(\mathcal{C}, \mathcal{E})$, whose right adjoint must send the terminal object $\beta \in[\mathcal{C}$, Set $]$ to a terminal object $\Delta \beta \in \mathrm{FC}(\mathcal{C}, \mathcal{E})$. As $\mathcal{C}$ and $\mathcal{E}$ are extensive, it follows from this and Lemma 19 that $\mathrm{FC}(\mathcal{C}, \mathcal{E}) \cong[\mathcal{C}, \mathcal{E}] / \mathrm{sc} \Delta \beta$ where to the right we have the full subcategory of the slice category on the sum-cartesian transformations; recall that sum-cartesian means that the naturality squares at coproduct coprojections are pullbacks.

Using (5.1) we have, like before, an equivalence $[\mathcal{C}, \mathcal{E}] / \Delta \beta \simeq[\mathrm{el} \beta, \mathcal{E}] \cong\left[\mathcal{U}_{\mathcal{E}}, \mathcal{E}\right]$; and, like before, an object $p: E \rightarrow \Delta \beta$ to the left is sum-cartesian just when the corresponding $\tilde{E} \in\left[\mathcal{U} \mathcal{E}_{\mathcal{e}}, \mathcal{E}\right]$ inverts the class $\mathcal{M}_{\Sigma}$ of continuous coproduct coprojections. Thus $\mathrm{FC}(\mathcal{C}, \mathcal{E}) \cong[\mathcal{C}, \mathcal{E}] / \mathrm{sc}_{\mathrm{s}} \Delta \beta \simeq\left[\mathcal{U} \mathcal{F}_{\mathcal{C}}, \mathcal{E}\right]$ as desired.

It remains to show that the two directions of the equivalence are given as in (3.6) and (3.7). In the latter case this is clear from the construction using (5.1). In the other direction, if $A \in \mathrm{FC}(\mathcal{C}, \mathcal{E})$, then the corresponding $\tilde{A} \in\left[\mathcal{U} \mathcal{E}_{\mathcal{C}}, \mathcal{E}\right]$ has 
its value at $(X, \mathcal{U})$ given by the pullback to the left in:
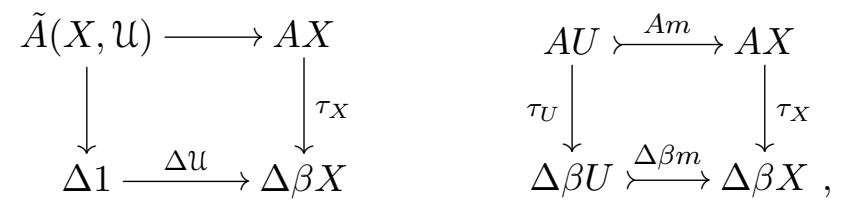

where $\tau: A \rightarrow \Delta \beta$ is induced by terminality of $\Delta \beta$ in $\mathrm{FC}(\mathcal{C}, \mathcal{E})$. Note, however, that $\mathcal{U}: 1 \rightarrow \beta X$ in Set is the meet of the subobjects $(\beta U \vdash \beta X)_{U \in \mathcal{U}}$. Since $\Delta$ is a right adjoint, it preserves meets, as does pullback along $\tau_{X}$; consequently, $\tilde{A}(X, \mathcal{U})$ is the meet of the subobjects $\left(\tau_{X}^{-1}(\Delta \beta U) \longmapsto A X\right)_{U \in \mathcal{U}}$. But since $\tau$ is sum-cartesian by Lemma 19, the square right above is a pullback for any $m: U \longmapsto X$ in $\mathcal{U}$, and so we conclude that $\tilde{A}$ is given as in (3.6) by

$$
\tilde{A}(X, \mathcal{U}) \cong \bigcap_{U \in \mathcal{U}} A U \subseteq A X .
$$

5.2. Ultramatrices, ultraspans and the relation to indexed sums. We now wish to describe how this result recaptures the indexed sum of ultrafilters. In fact, we will do something slightly more general to draw as perfect an analogy as possible with Proposition 16. The first step there was to transport the strict monoidal structure on the category FC(Set, Set) to obtain a monoidal structure on the equivalent [UFF, Set]. The analogue here is to transport the compositional structure of a 2-category of finite-coproduct-preserving functors along equivalences of each of its hom-categories to obtain an equivalent bicategory [4].

Definition 27. (i) The 2-category $\mathrm{FC}_{\text {Set }}$ has sets $X, Y, Z, \ldots$ as objects; homcategories given by $\mathrm{FC}_{\mathcal{S e t}}(X, Y)=\mathrm{FC}\left(\operatorname{Set}^{X}\right.$, $\left.\operatorname{Set}^{Y}\right)$; and composition given by the usual composition of functors and natural transformations.

(ii) The bicategory UEsp of ultrafilter species has sets as objects; hom-categories $\mathrm{UEsp}(X, Y)=\left[\mathcal{U F}_{\mathcal{S e t}^{X}}, \operatorname{Set}^{Y}\right]$; and composition obtained from that of $\mathrm{FC}_{\text {Set }}$ by transporting across the equivalences $\mathrm{FC}\left(\operatorname{Set}^{X}, \operatorname{Set}^{Y}\right) \simeq\left[\mathcal{U} \mathcal{F}_{\text {Set }^{X}}, \operatorname{Set}^{Y}\right]$.

The nomenclature "ultrafilter species" echoes Joyal's notion of a species of structures (espéces de structures [28]), and its generalisation in [16] to a bicategory Esp of generalised species of structures. We will not labour the comparison, but suffice it to say that in both bicategories, composition is given by a substitution formula, which in the case of Esp is given by equation (9) of [16], and for UEsp is given by a suitable generalisation of (3.8).

In Proposition 16, we reconstructed the tensor product of ultrafilters by

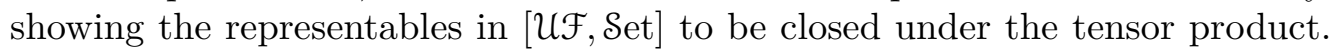
To reconstruct the indexed sum of ultrafilters, we will similarly show that pointwise representable 1-cells in UEsp are closed under composition. Here, $F \in \operatorname{UEsp}(X, Y)=\left[\mathcal{U F}_{\mathcal{S e t}^{X}}, \operatorname{Set}^{Y}\right]$ is pointwise representable if each functor $F(-)(y): \mathcal{U} \mathcal{F}_{\text {Set }} X \rightarrow$ Set is representable. The subcategory of pointwise representable functors is equivalent (via pointwise Yoneda) to the category $\left(\mathcal{U} \mathcal{F}_{\text {Set }^{X}}\right)^{Y}$, and so a typical pointwise representable 1-cell is presented by a $Y$-indexed family of pairs $\left(M_{y} \in \operatorname{Set}^{X}, \mathcal{U}_{y} \in \beta\left(X . M_{y}\right)\right)$. In fact, we prefer to think of these data in either one of the following two alternative ways. 
Definition 28. Let $X$ and $Y$ be sets.

(i) An ultramatrix from $X$ to $Y$ is a pair $(M, \mathcal{U})$ composed of a matrix of sets $M \in \operatorname{Set}^{X \times Y}$ together with a $Y$-indexed family of ultrafilters $\mathcal{U}_{y}$ on each column sum $M_{y}:=(\Sigma x \in X) M(x, y)$.

(ii) An ultrafamily $(g, \mathcal{U}): M \rightsquigarrow Y$ is a function $g: M \rightarrow Y$ together with an ultrafilter $\mathcal{U}_{y}$ on each fibre $g^{-1}(y)$. An ultraspan from $X$ to $Y$ is a span with left leg a function and right leg an ultrafamily:<smiles>[Y][Y4]([Y])=[V]</smiles>

It is easy to see using (2.1) that both ultramatrices and ultraspans from $X$ to $Y$ correspond to pointwise representables in $\operatorname{UEsp}(X, Y)$, and so to certain finitecoproduct-preserving functors $\operatorname{Set}^{X} \rightarrow \operatorname{Set}^{Y}$. As in the introduction, we may call these generalised ultraproduct functors. Using Proposition 24, we see that, one the one hand, the generalised ultraproduct functor $\operatorname{Set}^{X} \rightarrow \operatorname{Set}^{Y}$ encoded by the ultramatrix $(M, \mathcal{U})$ is given by:

$$
(H(x) \mid x \in X) \quad \mapsto \quad\left(\left(\Pi_{\mathcal{U}_{y}}(x, m) \in M_{y}\right) H(x) \mid y \in Y\right) .
$$

On the other hand, the ultraspan $(f,(g, \mathcal{U})): X \rightarrow Y$ encodes the functor

$$
\text { Set }^{X} \stackrel{\text { Set }^{f}}{\longrightarrow} \operatorname{Set}^{M} \stackrel{\Pi_{(g, u)}}{\longrightarrow} \operatorname{Set}^{Y}
$$

where $\Pi_{(g, \mathcal{}}$ is given by "ultraproduct on each fibre"; i.e., its $y$-component is given by restriction $\mathcal{S e t}^{M} \rightarrow \mathcal{S e t}^{g^{-1} y}$ followed by ultraproduct $\Pi_{\mathcal{U}_{y}}: \mathcal{S e t}^{g^{-1} y} \rightarrow$ Set.

The next two definitions are intended to describe how pointwise representable 1-cells in UEsp compose in terms of the representing ultramatrices or ultraspans.

Definition 29. If $(M, \mathcal{U})$ and $(N, \mathcal{V})$ are ultramatrices from $X$ to $Y$ and from $Y$ to $Z$, then their composition is the ultramatrix $(N \cdot M, \mathcal{V} \cdot \mathcal{U})$ from $X$ to $Z$ whose first component is given by the usual matrix multiplication:

$$
(N \cdot M)(x, z)=(\Sigma y \in Y)(N(y, z) \times M(x, y)) .
$$

As for the second component, note that for each $z \in Z$ we have an isomorphism

$$
\left(\Sigma(y, n) \in N_{z}\right) M_{y} \cong(N \cdot M)_{z}
$$

sending $(y, n, x, m)$ to $(x, y, n, m)$. We can therefore define the ultrafilter $(\mathcal{V} \cdot \mathcal{U})_{z}$ on $(N \cdot M)_{z}$ to be the transport across (5.4) of the ultrafilter on $\left(\Sigma(y, n) \in N_{z}\right) M_{y}$ given by the indexed sum $\left(\Sigma_{\mathcal{V}_{z}}(y, n) \in N_{z}\right) \mathcal{U}_{y}$.

Definition 30. Given ultraspans $(f,(g, \mathcal{U})): X \rightarrow Y$ and $(h,(k, \mathcal{V})): Y \rightarrow Z$, their composition is the ultraspan whose legs are given by the outer composites in:

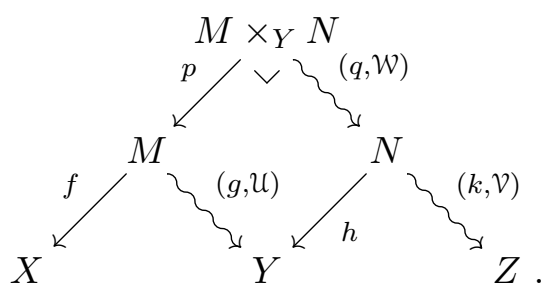


Here, $p$ and $q$ constitute a pullback of $g$ and $h$ in Set. To the top right, the pullback ultrafamily $(q, \mathcal{W}): M \times_{Y} N \rightsquigarrow N$ has $\mathcal{W}_{n}$ given by the transport of $\mathcal{U}_{h n}$ across the isomorphism $g^{-1}(h n) \cong q^{-1}(n)$. Finally, the composite $(k q, \mathcal{V W})$ of the ultrafamilies $(q, \mathcal{W})$ and $(k, \mathcal{V})$ has $(\mathcal{V W})_{z}$ given by the transport of $\left(\Sigma_{\mathcal{V}_{z}} n \in k^{-1} z\right) \mathcal{W}_{n}$ across the isomorphism $\left(\Sigma n \in k^{-1} z\right) q^{-1} n \cong(k q)^{-1} z$.

The validity of these descriptions is confirmed by:

Proposition 31. The pointwise representable 1-cells in UEsp are closed under composition, with the induced composition on ultramatrices and ultraspans given as in Definition 29 and Definition 30 respectively.

Proof. The identity 1-cells in UEsp are easily seen to be pointwise representable. As for binary composition, the composition laws in Definitions 29 and 30 correspond under (2.1), so that it suffices to check the claim on ultramatrices. So let $F \in \operatorname{UEsp}(X, Y)$ and $G \in \operatorname{UEsp}(Y, Z)$ be represented by the respective ultramatrices $(M, \mathcal{U})$ and $(N, \mathcal{V})$. By (5.3), the corresponding generalised ultraproduct functors $\int F \in \mathrm{FC}\left(\mathcal{S e t}^{X}\right.$, $\left.\operatorname{Set}^{Y}\right)$ and $\int G \in \mathrm{FC}\left(\operatorname{Set}^{Y}, \operatorname{Set}^{Z}\right)$ have respective actions on objects

$\left(\int F\right)(H)(y)=\left(\Pi_{\mathcal{U}_{y}}(x, m) \in M_{y}\right) H(x)$ and $\left(\int G\right)(K)(z)=\left(\Pi_{\mathcal{V}_{z}}(y, n) \in N_{z}\right) K(y)$. Therefore $\int G F \cong \int G \circ \int F: \operatorname{Set}^{X} \rightarrow \operatorname{Set}^{Z}$ satisfies

$$
\begin{aligned}
\left(\int G F\right)(H)(z) & \cong\left(\Pi_{\mathcal{V}_{z}}(y, n) \in N_{z}\right)\left(\Pi_{\mathcal{U}_{y}}(x, m) \in M_{y}\right) H(x) \\
& \cong\left(\Pi_{\left(\Sigma_{\mathcal{V}_{z}}(y, n) \in N_{z}\right) \mathcal{u}_{y}}(y, n, x, m) \in\left(\Sigma(y, n) \in N_{z}\right) M_{y}\right) H(x) \\
& \cong\left(\Pi_{(\mathcal{V} \cdot \mathcal{U})_{z}}(x, y, n, m) \in(N \cdot M)_{z}\right) H(x),
\end{aligned}
$$

using Proposition 9 and the definition of $(\mathcal{V} \cdot \mathcal{U})_{z}$. Thus, by (5.3) again, the pointwise representability of $G F$ is witnessed by the ultramatrix $(N \cdot M, \mathcal{V} \cdot \mathcal{U})$.

It follows from this result that there are bicategories UMtx (resp., US) in which objects are sets; 1-cells are ultramatrices (resp., ultraspans) composing as in Definition 29 (resp., Definition 30); and 2-cells are determined by the requirement that each bicategory be biequivalent to the locally full sub-bicategory of UEsp on the pointwise representable 1-cells.

It remains to show that the composition laws in UMtx and US allow us to reconstruct the indexed sum of ultrafilters, so fulfilling the objective of this section. This is easiest to see in the case of US. Suppose that we are given a set $X$ equipped with an ultrafilter $\mathcal{U}$ and an $X$-indexed family of sets $Y(x)$ each equipped with an ultrafilter $\mathcal{V}(x)$. We can represent these data as a pair of composable ultraspans as to the left in:<smiles>[Y][Y][Te][Y]([Y])[Y]</smiles><smiles>[Y][V][Al][V]</smiles>

whose composite encodes the indexed sum $\mathcal{U} . \mathcal{V}$ as right above.

Remark 32. In Remark 17 above, we explained how categories enriched over the monoidal category [ $\mathcal{H F}$, Set] can be thought of as ordinary categories endowed 
with abstract ultrapower functors. It is possible to extend this so as to capture categories endowed with abstract ultraproduct functors by using the theory of categories enriched in a bicategory as in [47].

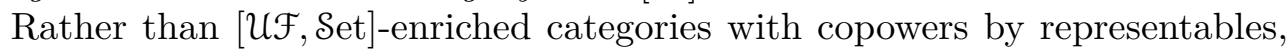
we consider UEsp-enriched categories with copowers by pointwise representable 1-cells. We might guess that such enriched categories correspond to ordinary categories $\mathcal{C}$ admitting abstract ultraproduct functors $\Pi_{\mathcal{U}}: \mathcal{C}^{X} \rightarrow \mathcal{C}$. The reality is slightly more subtle; while some details still require sorting out, it appears that the UEsp-enriched categories with copowers as above correspond to Set-indexed prestacks - i.e., pseudo-functors $\mathbb{C}:$ Set ${ }^{\text {op }} \rightarrow$ CAT satisfying a descent conditionequipped with suitably coherent abstract ultrapower functors $\Pi_{(f, \mathcal{})}: \mathbb{C}_{X} \rightarrow \mathbb{C}_{Y}$ for each ultrafamily $(f, \mathcal{U}): X \rightsquigarrow Y$.

While the details must await a further paper, these observations draw an interesting link to Makkai's ultracategories [40,41]. As in the introduction, an ultracategory is a category endowed with abstract ultraproduct structure, as well as interpretations for any ultramorphism, i.e., "definable map between ultraproducts". Makkai's main result is that the ultracategory structure on the category of models of a coherent theory $\mathbb{T}$ in either intuitionistic or classical first-order logic is sufficient to reconstruct $\mathbb{T}$ to within Morita equivalence; more precisely, it suffices to reconstruct the classifying pretopos of $\mathbb{T}$.

These results of Makkai were given new proofs by Lurie in [35], with a significantly simplified definition of what it means for an ultracategory to admit interpretations of any ultramorphism; and although we have not yet completed the analysis, it seems that this additional structure is exactly what UEsp-enrichment provides besides the existence of abstract ultraproduct functors. In future work we hope to investigate this further with a view to giving a purely enriched-categorical proof of Makkai's reconstruction result.

\section{LOCALLY CONNECTED CLASSIFYING TOPOSES}

In Section 4.3, we discussed the algebraic semantics for classical propositional theories in Boolean algebras, and for first-order theories in Boolean pretoposes. There are similar semantics for certain fragments of intuitionistic logic: in particular, the coherent fragment, which allows only the logical connectives $\exists, \vee, \wedge, \top, \perp$, has an algebraic semantics in distributive lattices (for the propositional case) and in pretoposes (for the first-order case); see, for example [42].

Now for classical propositional theories, there is a restricted semantics valued not in Boolean algebras but complete atomic Boolean algebras. Rather than the Lindenbaum-Tarski algebra $B_{\mathbb{P}}$, the appropriate universal model in this context is the power-set algebra $\mathcal{P}\left(T_{\mathbb{P}}\right)$ on the set $T_{\mathbb{P}}$ of complete theories extending $\mathbb{P}$, with the universal valuation of $\mathbb{P}$ in $\mathcal{P}\left(T_{\mathbb{P}}\right)$ sending each primitive proposition $\varphi$ to the set of complete extensions which validate it. Since $T_{\mathbb{P}}$ is equally the set $\beta\left(B_{\mathbb{P}}\right)$ of ultrafilters on $B_{\mathbb{P}}$, the passage from $B_{\mathbb{P}}$ to $\mathcal{P}\left(T_{\mathbb{P}}\right)$ can be understood in terms of the lattice-theoretic construction of canonical extension [27]: in general, for a Boolean algebra $B$, its canonical extension is the power-set algebra $B^{\delta}:=\mathcal{P}(\beta B)$.

Similarly, for coherent intuitionistic propositional theories, the semantics in distributive lattices restricts to one valued in completely distributive algebraic 
lattices; these are equally well the down-set lattices of posets, and posets are the same thing as symmetric, transitive Kripke frames, so this is really another view on the Kripke semantics of intuitionistic logic [46, §2.5]. In this context, the classifying distributive lattice $D_{\mathbb{P}}$ of a theory $\mathbb{P}$ is replaced by its canonical extension $D_{\mathbb{P}}^{\delta}$, constructible as in [21] as the downset-lattice of the poset of prime filters in $D_{\mathbb{P}}$ (ordered by reverse inclusion); this is an algebraic reformulation of the construction of the canonical Kripke model [46, Definition 2.6.4] of an intuitionistic propositional theory.

An obvious question is whether the alternate semantics detailed above lift from propositional to first-order logic. A variety of positive answers have been given to this question. One positive answer is given in [39]; there, Makkai introduces the notion of a prime-generated topos, as a Grothendieck topos whose subobject lattices are completely distributive algebraic ${ }^{1}$. He then considers semantics for a coherent intuitionistic first-order theory $\mathbb{T}$ valued in prime-generated toposes, and constructs the universal such model in what he calls the topos of types of $\mathbb{T}$. This is obtained from the classifying pretopos $\mathcal{C}=\mathcal{C l}(\mathbb{T})$ of $\mathbb{T}$ by a "categorified canonical extension", obtained by first forming a category $\mathcal{P} \mathcal{F}_{\mathcal{C}}$ of prime filters in $\mathcal{C}$, defined similarly to our $\mathcal{U F}_{\mathcal{C}}$, and then taking a suitable sheaf subcategory $\tau(\mathcal{C l}(\mathbb{T}))$ of $\left[\mathcal{P} \mathcal{F}_{\mathfrak{C}}^{\text {op }}\right.$, Set $]$. The name "topos of types" for $\tau(\mathfrak{C l}(\mathbb{T}))$ derives from the fact that, as Makkai puts it, it gives 'a reasonable codification of the 'discrete' (non topological) syntactical structure of types of the theory" [38, p.196]. The idea that the passage $\mathcal{C} l(\mathbb{T}) \mapsto \tau(\mathcal{C l}(\mathbb{T}))$ should be seen as a kind of canonical extension was made precise by Coumans in [13].

In this section, we take a slightly different view on lifting canonical extension to the first-order coherent context. In Makkai's approach, the distributive subobject lattices of $\mathrm{Cl}(\mathbb{T})$ are completed via canonical extension to the completely distributive algebraic subobject lattices of $\tau(\mathcal{C l}(\mathbb{T}))$. For us, the focus will instead be on the Boolean algebras of coproduct summands in $\mathrm{Cl}(\mathbb{T})$ - corresponding logically to decidable predicates in $\mathbb{T}$-which will be completed via canonical extension to complete atomic Boolean algebras of coproduct-summands. The property of having complete atomic Boolean algebras of coproduct-summands is, by [3, Theorem 15], characteristic of locally connected Grothendieck toposes, and so what we aim to describe in the locally connected classifying topos of a given pretopos $\mathcal{C}$. The existence of this follows from results of [18], but the description there is rather inexplicit; we aim to give a concrete construction in terms of sheaves on the category of ultrafilters, and to compare this to the toposes of types of Makkai and others.

6.1. The lextensive case. In this section, as a warm-up to our main result, we construct the locally connected classifying topos of a small lextensive categorythat is, a category which is both finitely complete and extensive.

We first make precise what we mean by this. Recall that a geometric morphism $f: \mathcal{E} \rightarrow \mathcal{F}$ between toposes is an adjoint pair of functors $f^{*} \dashv f_{*}: \mathcal{E} \rightarrow \mathcal{F}$ such that $f^{*}$ (the inverse image functor) preserves finite limits. We write

\footnotetext{
${ }^{1}$ This is not the original definition of prime-generation from [38], but an equivalent one from $[2, \S 3]$.
} 
LCGTop for the 2-category of locally connected Grothendieck toposes, geometric morphisms and natural transformations $f^{*} \Rightarrow g^{*}$, and write Lext for the 2category of lextensive categories, lextensive functors (i.e., ones preserving finite limits and finite coproducts) and arbitrary natural transformations. As every locally connected Grothendieck topos and every inverse image functor between such is lextensive, we have a forgetful 2-functor LCGTop $^{\text {op }} \rightarrow$ Lext.

Definition 33. A locally connected classifying topos for an extensive category $\mathcal{C}$ is a left biadjoint at $\mathcal{C}$ for the forgetful 2 -functor LCGTop $^{\text {op }} \rightarrow$ Lext.

Here, and in what follows, when we speak of a left biadjoint at $X$ for a 2 -functor $U: \mathrm{A} \rightarrow \mathrm{B}$, we mean a birepresentation (in the sense of [45]) for the 2-functor $\mathrm{B}\left(X, U_{-}\right): \mathrm{A} \rightarrow$ CAT. More concretely, then, a locally connected classifying topos for the lextensive $\mathcal{C}$ comprises a locally connected Grothendieck topos $\mathcal{L} \mathrm{c}(\mathcal{C})$ and a lextensive functor $\eta: \mathcal{C} \rightarrow \mathcal{L} \mathrm{c}(\mathcal{C})$ which is universal in the sense that, for each locally connected Grothendieck topos $\mathcal{E}$, we have an equivalence:

$$
\operatorname{LCGTop}(\mathcal{E}, \mathcal{L} \mathrm{c}(\mathcal{C})) \simeq \operatorname{Lext}(\mathcal{C}, \mathcal{E})
$$

induced by the assignment $f \mapsto f^{*} \circ \eta$.

Our goal is to give an explicit construction of a locally connected classifying topos for any small lextensive category $\mathcal{C}$. For this, we require the result sometimes known as Diaconescu's theorem; it can be found proved in, for example, [36, Theorem VII.7.2].

Proposition 34. If $\mathcal{A}$ is a small category, then the presheaf topos [ $\mathcal{A}^{\mathrm{op}}$, Set] classifies flat functors out of $\mathcal{A}$. More precisely, for each Grothendieck topos $\mathcal{E}$, the assignment $f \mapsto f^{*} \circ y$ induces an equivalence of categories

$$
\operatorname{GTop}\left(\mathcal{E},\left[\mathcal{A}^{\mathrm{op}}, \operatorname{Set}\right]\right) \simeq \operatorname{Flat}(\mathcal{A}, \mathcal{E}) .
$$

Here, we define Flat $(\mathcal{A}, \mathcal{E})$ as the full subcategory of $[\mathcal{A}, \mathcal{E}]$ on the flat functors, but for this we should clarify what "flat" means. One definition is that $F: \mathcal{A} \rightarrow \mathcal{E}$ is flat just when $\operatorname{Lan}_{y} F:\left[\mathcal{A}^{\mathrm{op}}\right.$, Set $] \rightarrow \mathcal{E}$, its left Kan extension along the Yoneda embedding of $\left[\mathcal{A}^{\mathrm{op}}, \mathcal{S e t}\right]$, preserves finite limits; this is a general categorical definition which makes sense for any small $\mathcal{A}$ and cocomplete $\mathcal{E}$. On the other hand, when $\mathcal{E}$ is a Grothendieck topos as above, a more explicit characterisation is possible which generalises a well-known characterisation when $\mathcal{E}=$ Set.

Given $F \in[\mathcal{A}, \mathcal{E}]$, we write el $F$ for the category of elements of $F$ : the internal category in $\mathcal{E}$ with underlying graph

$$
\sum_{a, b \in \mathcal{A}} \sum_{f \in \mathcal{A}(a, b)} D a \stackrel{s}{\underset{t}{\longrightarrow}} \sum_{a \in \mathcal{A}} D a
$$

where $s$ maps the $(a, b, f)$-summand to the $a$-summand via $1_{D a}$, and where $t$ maps the $(a, b, f)$-summand to the $b$-summand via $D f$. There is a standard notion - see, for example [25, Definition B2.6.2] - of what it means for an internal category $\mathbb{C}$ in a topos to be cofiltered; in the internal language of the topos, it says that "every finite diagram in $\mathbb{C}$ has a cocone under it". The key result we will need is the following; for a proof, see [25, Theorem B3.2.7]. 
Proposition 35. If $\mathcal{A}$ is a small category and $\mathcal{E}$ is a Grothendieck topos, then $F: \mathcal{A} \rightarrow \mathcal{E}$ is flat if and only if the internal category el $F$ in $\mathcal{E}$ is cofiltered.

With these preliminaries in place, we can now give:

Proposition 36. If $\mathcal{C}$ is small and lextensive, then [ $\mathcal{F}_{\mathcal{C}}{ }^{\text {op }}$, Set] is a locally connected classifying topos for $\mathcal{C}$.

Proof. Like any presheaf topos, $\left[\mathcal{U F}_{\mathcal{e}}{ }^{\text {op }}\right.$, Set] is locally connected. For the classifying property, we must exhibit equivalences $\operatorname{LCGTop}\left(\mathcal{E},\left[\mathcal{U} \mathcal{F}_{\mathcal{e}}{ }^{\text {op }}, \operatorname{Set}\right]\right) \simeq \operatorname{Lext}(\mathcal{C}, \mathcal{E})$, pseudonaturally in $\mathcal{E}$, which we will do by composing pseudonatural equivalences:

$$
\operatorname{LCGTop}\left(\mathcal{E},\left[\mathcal{U} \mathcal{F}_{\mathcal{e}}{ }^{\mathrm{op}}, \operatorname{Set}\right]\right) \stackrel{\simeq}{\longrightarrow} \operatorname{Flat}\left(\mathcal{U} \mathcal{F}_{\mathcal{C}}, \mathcal{E}\right) \stackrel{\simeq}{\longrightarrow} \operatorname{Lext}(\mathcal{C}, \mathcal{E}) \text {. }
$$

The first of these is (6.2). As for the second, we have by Theorem 26 that

$$
\left[\mathcal{U} \mathcal{F}_{\mathcal{C}}, \mathcal{E}\right] \simeq \mathrm{FC}(\mathcal{C}, \mathcal{E})
$$

for any locally connected Grothendieck topos $\mathcal{E}$, and by considering the explicit formula (3.7) for the rightward direction, we see that these equivalences are pseudonatural in inverse image functors. We will thus have the desired pseudonatural equivalence if we can show that, in (6.4), the flat functors on the left-hand side correspond to the finite-limit-preserving ones on the right.

Towards this goal, we recall from Definition 21 the category $\mathcal{U} \mathcal{E}_{\mathfrak{e}}$ of which $\mathcal{U} \mathcal{F}_{\mathfrak{e}}$ is a localisation, and consider the span $\pi: \mathcal{C} \leftarrow \mathcal{U} \mathcal{E}_{\mathcal{C}} \rightarrow \mathcal{U} \mathcal{F}_{\mathcal{C}}: \iota$ whose two legs are the forgetful functor and the localisation functor respectively. It is easy to see from the formula (3.7) that the left-to-right direction of (6.4) sends $B: \mathcal{U} \mathcal{F}_{\mathcal{C}} \rightarrow \mathcal{E}$ to its image under the composite functor

$$
\left[\mathcal{U F} \mathcal{F}_{\mathcal{C}}, \mathcal{E}\right] \stackrel{\iota^{*}}{\longrightarrow}\left[\mathcal{U} \mathcal{E}_{\mathcal{e}}, \mathcal{E}\right] \stackrel{\operatorname{Lan}_{\pi}}{\longrightarrow}[\mathcal{C}, \mathcal{E}] .
$$

It therefore suffices to prove that:

(i) $B: \mathcal{U} \mathcal{F}_{\mathcal{e}} \rightarrow \mathcal{E}$ is flat if and only if $B \iota: \mathcal{U} \mathcal{E}_{\mathcal{e}} \rightarrow \mathcal{E}$ is flat. We saw above that $\mathcal{U} \mathcal{F}_{\mathcal{C}} \cong \mathcal{U} \mathcal{E}_{\mathcal{C}}\left[\mathcal{M}_{\Sigma}^{-1}\right]$, the localisation at the class of continuous coproduct coprojections. Since $\mathcal{M}_{\Sigma}$ is a pullback-stable, composition-closed class of arrows, there is (for example by [26, Proposition C2.1.9]) a Grothendieck topology $J$ on $\mathcal{U} \mathcal{E}_{e}$ whose covering sieves are those which contain any map in $\mathcal{M}_{\Sigma}$. The condition for a functor $F: U \mathcal{E}_{e}^{\text {op }} \rightarrow$ Set to be a $J$-sheaf is now precisely the condition that it inverts each $m \in \mathcal{M}_{\Sigma}$, and so we may identify [ $\mathcal{U F}_{\mathcal{C}}{ }^{\text {op }}$, Set] with $\mathcal{S h}\left(\mathcal{U} \mathcal{E}_{\mathcal{C}}\right)$, and the sheafification adjunction with the adjunction $\operatorname{Lan}_{\iota} \dashv$ $\iota^{*}:\left[\mathcal{U} \mathcal{F}_{\mathcal{C}}{ }^{\text {op }}, \mathcal{S e t}\right] \rightarrow\left[\mathcal{U} \mathcal{E}_{\mathcal{C}}{ }^{\text {op }}\right.$, Set $]$; so in particular, $\operatorname{Lan}_{\iota}$ preserves finite limits. We now use this to prove the claim. Note that $\operatorname{Lan}_{y}(B \iota) \cong\left(\operatorname{Lan}_{y} B\right) \circ \operatorname{Lan}_{\iota}$, so that if $B$ is flat, then so too is $B \iota$. On the other hand, since $\operatorname{Lan}_{\iota} \circ \iota^{*} \cong 1$, we have that $\operatorname{Lan}_{y} B \cong \operatorname{Lan}_{y}(B \iota) \circ \iota^{*}$ so that if $B \iota$ is flat then so too is $B$.

(ii) $F: \mathcal{U} \mathcal{E}_{\mathcal{C}} \rightarrow \mathcal{E}$ is flat if and only if $\operatorname{Lan}_{\pi} F: \mathcal{C} \rightarrow \mathcal{E}$ preserves finite limits. Since the value of $\operatorname{Lan}_{\pi} F: \mathcal{C} \rightarrow \mathcal{E}$ at $X$ is $\sum_{\mathcal{U} \in \beta X} F(X, \mathcal{U})$, it is an easy calculation to see that the internal categories el $F$ and el $\left(\operatorname{Lan}_{\pi} F\right)$ are isomorphic. So $F$ is flat if and only if $\operatorname{Lan}_{\pi} F$ is flat. But since $\mathcal{C}$ admits all finite limits, $\operatorname{Lan}_{\pi} F$ is flat if and only if it is finite-limit-preserving; see, for example [25, Lemma B3.2.5].

By tracing the identity geometric morphism on $\left[\mathcal{U F}_{\mathcal{C}}{ }^{\text {op }}\right.$, Set $]$ through this proof, we see that the universal lextensive functor $\eta: \mathcal{C} \rightarrow\left[\mathcal{U F}_{\mathcal{C}}{ }^{\mathrm{op}}\right.$, Set $]$ is the image 
under (3.7) of the Yoneda embedding $\mathcal{U} \mathcal{F}_{\mathcal{C}} \rightarrow\left[\mathcal{U F}_{\mathcal{C}}{ }^{\text {op }}\right.$, Set $]$, and so given by:

$$
\eta(X)=\sum_{u \in \beta X} y_{(X, \mathcal{u})} .
$$

6.2. The pretopos case. Let us now write Pretop for the 2-category of pretoposes, pretopos morphisms and all natural transformations. Like before, every locally connected Grothendieck topos is a pretopos and every inverse image functor is a pretopos morphism, so that we have a forgetful 2-functor LCGTop $^{\text {op }} \rightarrow$ Pretop.

Definition 37. A locally connected classifying topos for a pretopos $\mathcal{C}$ is a left biadjoint at $\mathcal{C}$ for the forgetful 2-functor LCGTop $^{\text {op }} \rightarrow$ Pretop.

It is known that every small pretopos $\mathcal{C}$ has a locally connected classifying topos. To see this, we factor the forgetful 2-functor of the preceding definition as the composite of the two forgetful 2-functors

$$
\text { LCGTop }^{\text {op }} \rightarrow \text { GTop }^{\text {op }} \rightarrow \text { Pretop }
$$

viewing a locally connected Grothendieck topos as a Grothendieck topos, and a Grothendieck topos as a pretopos. The second factor is well-known to have a left biadjoint at every small pretopos $\mathcal{C}$, given by the topos of sheaves $\mathcal{S h}(\mathcal{C})$ for the topology of finite jointly epimorphic families. On the other hand, the first factor is known to have a left biadjoint given by the locally connected coclosure of [18]. It follows that the composite has a left biadjoint at every small pretopos.

One difficulty with the preceding argument is that the construction of the locally connected coclosure in [18] is inexplicit, relying at a crucial point on the adjoint functor theorem. Our objective in this section is to give a concrete description of the locally connected classifying topos of any small De Morgan pretopos. The notion of De Morgan pretopos is an obvious generalisation of the notion of De Morgan topos described, for example in [26, $\S \mathrm{D} 4.6]$. In giving the definition, we recall a pseudocomplement of an element $a$ in a distributive lattice is an element $\neg a$ which is disjoint from $a$, and is moreover the maximal such element; i.e., such that $a \wedge b=\perp$ if and only if $b \leqslant \neg a$.

Definition 38. A distributive lattice $A$ is a Stone algebra [22] if it admits all pseudocomplements and satisfies $\neg a \vee \neg \neg a=\top$ for all $a \in A$. A pretopos $\mathcal{C}$ is De Morgan if each subobject lattice $\operatorname{Sub}_{\mathfrak{C}}(X)$ is a Stone algebra.

An equivalent characterisation of a De Morgan pretopos is as one in which each inclusion of meet semi-lattices $\operatorname{Sum}_{\mathcal{C}}(X) \rightarrow \operatorname{Sub}_{\mathcal{C}}(X)$ has a left adjoint sending $A$ to $\neg \neg A$. The relevance of the condition to our investigations is isolated in the following result, whose significance will become clear shortly. In its proof, we use the operation $\exists_{f}: \operatorname{Sub}_{\mathcal{C}}(X) \rightarrow \operatorname{Sub}_{\mathcal{e}}(Y)$ of direct image along a map $f: X \rightarrow Y$ of a pretopos $\mathcal{C}$. This operation is left adjoint to pullback $f^{-1}: \operatorname{Sub}_{\mathcal{C}}(Y) \rightarrow \operatorname{Sub}_{\mathcal{C}}(X)$ and satisfies the Beck-Chevalley and Frobenius conditions; see [25, §A1.3].

Proposition 39. If $\mathrm{C}$ is a De Morgan pretopos, then $\mathcal{U F}_{\mathrm{e}}$ satisfies the right Ore condition: that is, each cospan in $\mathcal{U F}_{\mathcal{C}}$ as in the solid part of the following diagram 
can be completed to a commuting square as shown:

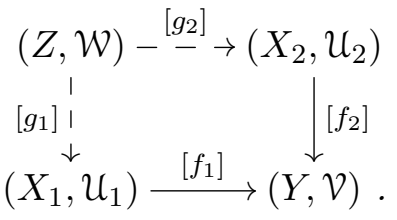

Proof. Since every map in $\mathcal{U F}_{\mathcal{C}}$ factors as an isomorphism followed by the equivalence class of a total map, we lose no generality in assuming that the $f_{i}$ 's in (6.5) are total. We can therefore form their pullback $g_{1}: X_{1} \leftarrow Z \rightarrow X_{2}: g_{2}$ in $\mathcal{C}$, and consider the subset $\mathcal{F} \subseteq \operatorname{Sum}_{\mathcal{C}}(Z)$ given as the upward closure of

$$
\left\{g_{1}^{-1}\left(U_{1}\right) \cap g_{2}^{-1}\left(U_{2}\right): U_{1} \in \mathcal{U}_{1}, U_{2} \in \mathcal{U}_{2}\right\} \text {. }
$$

This subset is easily a filter on $\operatorname{Sum}_{\mathcal{C}}(Z)$, and we claim it is a proper filter; thus, for any $U_{1} \in \mathcal{U}_{1}$ and $U_{2} \in \mathcal{U}_{2}$ we must show that $g_{1}^{-1}\left(U_{1}\right) \cap g_{2}^{-1}\left(U_{2}\right) \neq \perp$. Now, by Frobenius, Beck-Chevalley, and Frobenius we have

$$
\begin{aligned}
\exists_{f_{1} g_{1}}\left(g_{1}^{-1}\left(U_{1}\right) \cap g_{2}^{-1}\left(U_{2}\right)\right) & =\exists_{f_{1}}\left(U_{1} \cap \exists_{g_{1}}\left(g_{2}^{-1}\left(U_{2}\right)\right)\right) \\
& =\exists_{f_{1}}\left(U_{1} \cap f_{1}^{-1}\left(\exists_{f_{2}}\left(U_{2}\right)\right)\right) \\
& =\exists_{f_{1}}\left(U_{1}\right) \cap \exists_{f_{2}}\left(U_{2}\right),
\end{aligned}
$$

and so, since direct image preserves and reflects $\perp$, we must equally show that $\exists_{f_{1}}\left(U_{1}\right) \cap \exists_{f_{2}}\left(U_{2}\right) \neq \perp$. If we set $V_{i}=\neg \neg \exists_{f_{i}}\left(U_{i}\right)$ then, by standard properties of pseudocomplementation, this is in turn equivalent to showing that $V_{1} \cap V_{2} \neq \perp$. Since $\mathcal{C}$ is De Morgan, we have $V_{i} \in \operatorname{Sum}_{\mathcal{C}}(Y)$; moreover, $U_{i} \in \mathcal{U}_{i}$ and $U_{i} \subseteq f_{i}^{-1}\left(\exists_{f_{i}}\left(U_{i}\right)\right) \subseteq f_{i}^{-1}\left(V_{i}\right)$ implies $f_{i}^{-1}\left(V_{i}\right) \in \mathcal{U}_{i}$, and so $V_{i} \in \mathcal{V}$ since $\left(f_{i}\right)_{!}\left(\mathcal{U}_{i}\right)=\mathcal{V}$. Since $\mathcal{V}$ is an ultrafilter, we conclude that $V_{1} \cap V_{2} \neq \perp$ as desired.

This proves that (6.6) generates a proper filter $\mathcal{F}$. By the Boolean prime ideal theorem, we can extend this to an ultrafilter $\mathcal{W} \in \beta Z$, which by construction satisfies $\mathcal{U}_{i} \subseteq\left(g_{i}\right) !(\mathcal{W})$ for $i=1,2$, and so $\mathcal{U}_{i}=\left(g_{i}\right)_{!}(\mathcal{W})$ (since both sides are ultrafilters). We have thus completed (6.6) to a commuting square as desired.

The key to constructing the locally connected classifying topos of a small De Morgan pretopos is the following standard result on geometric morphisms into sheaf toposes proved, for example, in [36, Lemma VII.7.3]. In the statement, we write $\operatorname{CovFlat}(\mathcal{A}, \mathcal{E})$ for the category of flat functors $\mathcal{A} \rightarrow \mathcal{E}$ which are also cover-preserving, in the sense of sending covers to jointly epimorphic families.

Proposition 40. Let $\mathcal{A}$ be a small site and $i: \operatorname{Sh}(\mathcal{A}) \rightarrow\left[\mathcal{A}^{\mathrm{op}}\right.$, Set $]$ the associated inclusion of toposes. Under the equivalence (6.2), a geometric morphism $\mathcal{E} \rightarrow$ $\left[\mathcal{A}^{\mathrm{op}}\right.$, Set] factors through $i$ just when the corresponding flat functor $\mathcal{A} \rightarrow \mathcal{E}$ is cover-preserving. Consequently, (6.2) restricts back to an equivalence

$$
\operatorname{GTop}(\mathcal{E}, \operatorname{Sh}(\mathcal{A})) \simeq \operatorname{CovFlat}(\mathcal{A}, \mathcal{E}) .
$$

The locally connected classifying topos of the small De Morgan pretopos $\mathcal{C}$ will be obtained as a topos of sheaves on $\mathcal{U F}_{\mathcal{C}}$ for a suitable Grothendieck topology, and its universal property verified via a chain of pseudonatural equivalences 
$\operatorname{LCGTop}\left(\mathcal{E}, \operatorname{Sh}\left(\mathcal{U} \mathcal{F}_{\mathcal{C}}\right)\right) \simeq \operatorname{CovFlat}\left(\mathcal{U} \mathcal{F}_{\mathcal{C}}, \mathcal{E}\right) \simeq \operatorname{Pretop}(\mathcal{C}, \mathcal{E})$, each of whose terms is a restriction of the corresponding term in (6.3).

Since a pretopos morphism out of $\mathcal{C}$ is a lextensive functor which also preserves regular epimorphisms, the topology on $\mathcal{U} \mathcal{F}_{\mathcal{C}}$ must be chosen so that, under the equivalence $\operatorname{Flat}\left(\mathcal{U} \mathcal{F}_{\mathcal{C}}, \mathcal{E}\right) \simeq \operatorname{Lext}(\mathcal{C}, \mathcal{E})$ of $(6.3)$, the cover-preserving functors to the left correspond to the regular-epimorphism-preserving ones to the right.

We now describe such a topology, specifying it in terms of a coverage [26, Definition C2.1.1]; this involves assigning to each object $X$ a set of covering families $\left(f_{i}: X_{i} \rightarrow X \mid i \in I\right)$ satisfying the stability property:

(C) For any cover $\left(f_{i}: X_{i} \rightarrow X \mid i \in I\right)$ and any map $g: Y \rightarrow X$ in $\mathcal{A}$, there is a cover $\left(h_{j}: Y_{j} \rightarrow Y \mid j \in J\right)$ such that each $g h_{j}$ factors through some $f_{i}$.

Proposition 41. Let $\mathcal{C}$ be a pretopos. There is a coverage on $\mathcal{U F}_{\mathfrak{C}}$ for which a typical cover of the object $(Y, \mathcal{V}) \in \mathcal{U F}_{\mathfrak{C}}$ is of the form

$$
\langle f, \mathcal{V}\rangle:=\left([f]:(X, \mathcal{U}) \rightarrow(Y, \mathcal{V}) \mid \mathcal{U} \in \beta X, f_{!}(\mathcal{U})=\mathcal{V}\right)
$$

for any $f: X \rightarrow Y$ whose image $\operatorname{im} f \longmapsto Y$ is (a coproduct injection and) in $\mathcal{V}$.

Proof. We must verify condition $(\mathrm{C})$. So consider $\langle f, \mathcal{V}\rangle$ as above and a map $[g]:\left(Y^{\prime}, \mathcal{V}^{\prime}\right) \rightarrow(Y, \mathcal{V})$ in $\mathcal{U} \mathcal{F}_{\mathcal{C}}$ defined on some $m: V^{\prime} \longmapsto Y^{\prime}$ in $\mathcal{V}^{\prime}$. We first pull back $f$ along $g$ in $\mathcal{C}$ as in the left-hand square below, and now define $f^{\prime}=m q: X^{\prime} \rightarrow Y^{\prime}$. By assumption, $\operatorname{im} f \in \mathcal{V}$; since $g$ is continuous and image factorisations are pullback-stable, it follows that im $f^{\prime} \in \mathcal{V}^{\prime}$. Moreover, for each $\left[f^{\prime}\right]:\left(X^{\prime}, \mathcal{U}^{\prime}\right) \rightarrow\left(Y^{\prime}, \mathcal{V}^{\prime}\right)$ in $\left\langle f^{\prime}, \mathcal{V}^{\prime}\right\rangle$, the composite $\left[g f^{\prime}\right]$ factorises through a map in $\langle f, \mathcal{V}\rangle$ as to the right in:
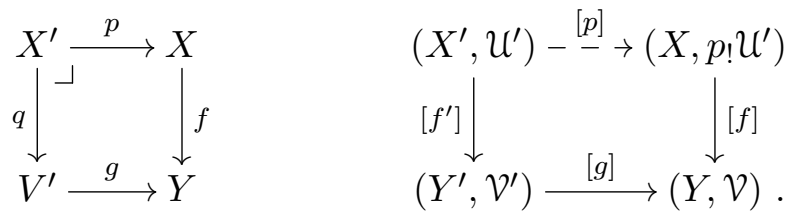

This proves that the covers do indeed satisfy condition $(\mathrm{C})$.

We write $\mathcal{S h}\left(\mathcal{U F}_{\mathcal{C}}\right)$ for the topos of sheaves on $\mathcal{U F}_{\mathcal{C}}$ for this coverage.

Theorem 42. Let $\mathcal{C}$ be a small De Morgan pretopos. The topos $\operatorname{Sh}\left(\mathcal{U F}_{\mathcal{C}}\right)$ is a locally connected classifying topos for $\mathrm{C}$, and is itself De Morgan.

Proof. We begin by showing that $\mathcal{S h}\left(\mathcal{U F}_{\mathcal{C}}\right)$ is locally connected and De Morgan. Since $\mathcal{C}$ is De Morgan, we know by Proposition 39 that $\mathcal{U} \mathcal{F}_{\mathcal{C}}$ satisfies the right Ore condition, and so by [26, Examples C3.3.11(a)] and [11, Corollary 2.8], the sheaf topos $\operatorname{Sh}\left(\mathcal{U} \mathcal{F}_{\mathcal{C}}\right)$ will be both locally connected and De Morgan so long as every covering family $\langle f, \mathcal{V}\rangle$ as in (6.8) is non-empty. Thus, given $\mathcal{V} \in \beta Y$ and $f: X \rightarrow Y$ in $\mathcal{C}$ with im $f \in \mathcal{V}$, we must show that there exists an ultrafilter $\mathcal{U} \in \beta X$ with $f_{!}(\mathcal{U})=\mathcal{V}$. Much as in Proposition 39, we consider the subset $\mathcal{F} \subseteq \operatorname{Sum}_{\mathcal{C}}(X)$ given as the upwards-closure of

$$
\left\{f^{-1}(V): V \in \mathcal{V}\right\} \text {. }
$$

Like there, $\mathcal{F}$ is a filter which we claim is moreover proper. Indeed, if $\perp=f^{-1}(V)$ for some $V \in \operatorname{Sum}_{\mathcal{C}}(Y)$, then also $\perp=\exists_{f}\left(f^{-1}(V) \cap \top\right)=V \cap \operatorname{im} f$ by Frobenius; 
whence $V \notin \mathcal{V}$ since $\operatorname{im} f \in \mathcal{V}$. Like before, we can now use the Boolean prime ideal theorem to find an ultrafilter $\mathcal{U} \subseteq \operatorname{Sum}_{\mathcal{C}}(X)$ extending $\mathcal{F}$ which, by construction, will satisfy $\mathcal{V} \subseteq f_{!}(\mathcal{U})$ and hence (since both are ultrafilters) $\mathcal{V}=f_{!}(\mathcal{U})$.

So $\mathcal{S h}\left(\mathcal{U} \mathcal{F}_{\mathcal{C}}\right)$ is locally connected and De Morgan; it remains to verify the classifying property, for which we must exhibit equivalences $\left.\operatorname{LCGTop}\left(\mathcal{E}, \mathcal{S h}\left(\mathcal{U} \mathcal{F}_{\mathcal{C}}\right), \mathcal{S e t}\right]\right) \simeq$ Pretop $(\mathcal{C}, \mathcal{E})$, pseudonaturally in $\mathcal{E}$. As discussed above, these will be obtained by composing pseudonatural equivalences:

$$
\operatorname{LCGTop}\left(\mathcal{E}, \mathcal{S h}\left(\mathcal{U} \mathcal{F}_{\mathcal{C}}\right)\right) \stackrel{\simeq}{\longrightarrow} \operatorname{CovFlat}\left(\mathcal{U F} \mathcal{F}_{\mathcal{C}}, \mathcal{E}\right) \stackrel{\simeq}{\longrightarrow} \operatorname{Pretop}(\mathcal{C}, \mathcal{E})
$$

of which the first is (6.7), and the second is obtained by restricting the right-hand equivalence $\operatorname{Flat}\left(\mathcal{U} \mathcal{F}_{\mathcal{e}}, \mathcal{E}\right) \simeq \operatorname{Lext}(\mathcal{C}, \mathcal{E})$ of $(6.3)$. The only point to check is that the cover-preserving functors to the left of this latter equivalence correspond to the regular-epimorphism-preserving ones to the right.

So suppose given a covering family $\langle f, \mathcal{V}\rangle$ as in (6.8). We may form the image factorisation $f=m e: X \rightarrow \operatorname{im} f \longmapsto Y$, and since by assumption $\operatorname{im} f \in \mathcal{V}$, we conclude that

$$
\langle f, \mathcal{V}\rangle=\left((X, \mathcal{U}) \stackrel{[e]}{\longrightarrow}\left(\operatorname{im} f,\left.\mathcal{V}\right|_{\operatorname{im} f}\right) \stackrel{[m]}{\longrightarrow}(Y, \mathcal{V})\right)_{\mathcal{U} \in \beta X, e_{!}(\mathcal{U})=\left.\mathcal{V}\right|_{\operatorname{im} f}} .
$$

Since $[m]$ is invertible in $\mathcal{U} \mathcal{F}_{\mathfrak{C}}$, this family will be sent to a jointly epimorphic one just when $\left\langle e,\left.\mathcal{V}\right|_{\operatorname{im} f}\right\rangle$ is; whence a functor $A: \mathcal{U} \mathcal{F}_{\mathcal{E}} \rightarrow \mathcal{E}$ preserves all covers just when it preserves ones $\langle f, \mathcal{V}\rangle$ as in (6.8) with $f$ a regular epimorphism. This is equally to say that, for each $f: X \rightarrow Y$ and each $\mathcal{V} \in \beta Y$, the map to the left in:

$$
\sum_{\substack{\mathcal{U} \in \beta X \\ f_{!}(\mathcal{U})=\mathcal{V}}} A(X, \mathcal{U}) \rightarrow A(Y, \mathcal{V}) \quad \sum_{\mathcal{U} \in \beta X} A(X, \mathcal{U}) \rightarrow \sum_{\mathcal{V} \in \beta X} A(Y, \mathcal{V})
$$

obtained by copairing the maps $A([f]): A(X, \mathcal{U}) \rightarrow A(Y, \mathcal{V})$ is an epimorphism in $\mathcal{E}$. Summing these left-hand maps over all $\mathcal{V} \in \beta Y$ and using infinite extensivity of $\mathcal{E}$, this is equally the condition that, for each $f: X \rightarrow Y$ in $\mathcal{C}$, the map right above is an epimorphism. Since this map is the value at $f$ of the functor $\int A: \mathcal{C} \rightarrow \mathcal{E}$ corresponding to $A$ under (3.7), this completes the proof.

Remark 43. As before, chasing the identity geometric morphism $\mathcal{S h}\left(\mathcal{U F}_{\mathcal{C}}\right) \rightarrow$ $\mathcal{S h}\left(\mathcal{U} \mathcal{F}_{\mathcal{C}}\right)$ through this proof shows that the universal pretopos map $\eta: \mathcal{C} \rightarrow \operatorname{Sh}\left(\mathcal{U} \mathcal{F}_{\mathcal{C}}\right)$ is the image under (3.7) of the composite ay: $\mathcal{U F}_{\mathcal{C}} \rightarrow \mathcal{S h}\left(\mathcal{U} \mathcal{F}_{\mathcal{C}}\right)$ of the Yoneda embedding and the sheafification functor. As such it is given by:

$$
\eta(X)=\sum_{u \in \beta X} a y_{(X, u)} .
$$

Now, each object $a y_{(X, \mathcal{u})}$ is connected in $\mathcal{S h}\left(\mathcal{U F}_{\mathcal{C}}\right)$, in the sense of having no nontrivial coproduct decomposition; and so it follows that $\operatorname{Sum}_{\mathcal{L F}}(\eta X)=\mathcal{P}(\beta X)$ is the canonical extension of the Boolean algebra $\operatorname{Sum}_{\mathcal{C}}(X)$. This justifies the link drawn in the introduction to this section between locally connected classifying toposes, and canonical extension for Boolean algebras.

Remark 44. We remarked above that the 2-functor GTop ${ }^{\text {op }} \rightarrow$ Pretop has a left biadjoint at every small pretopos given by the topos $\mathcal{S h}(\mathcal{C})$ of sheaves on $\mathcal{C}$ for the topology of finite jointly epimorphic families. The toposes arising in this way are commonly known as coherent toposes; moreover, by [11, Theorem 3.11], the 
coherent topos associated to a small De Morgan pretopos is itself De Morgan. Given this, another way of seeing Theorem 42 is as giving an explicit construction of the locally connected coclosure [18] of any coherent De Morgan topos.

One may reasonably ask if we have a similar explicit construction upon dropping the qualifier "coherent". The answer is yes, so long as we assume that every cardinal is smaller than some strongly compact cardinal. In this case, for any De Morgan Grothendieck topos $\mathcal{E}$, we can find a strongly compact cardinal $\kappa$ such that $\mathcal{E}$ is the free completion of a small De Morgan $\kappa$-ary pretopos - that is, a pretopos with pullback-stable $\kappa$-small coproducts. We can thus reduce the problem to constructing the locally connected classifying topos of a small $\kappa$-ary De Morgan pretopos; and we can do this by tracing through the definitions and results of this paper replacing everywhere finite coproducts by $\kappa$-small coproducts. The main change, as in [9], is that we must replace ultrafilters by $\kappa$-complete ultrafilters - ones closed under $\kappa$-small intersections. The assumption of strong compactness of $\kappa$ is needed in the proofs of Proposition 39 and Theorem 42, where we are now required to extend the $\kappa$-complete filters (6.6) and (6.9) to $\kappa$-complete ultrafilters.

6.3. Relation to toposes of types. In the introduction to this section, we have already discussed the analogies between our construction of a locally connected classifying topos, and Makkai's topos of types from [38]. We now provide a more detailed technical comparison between these constructions and other similar constructions in the literature.

The earliest "topos of types" in fact predates Makkai, appearing in Joyal and Reyes' [29]. Given a pretopos $\mathcal{C}$, a prime filter on $X \in \mathcal{C}$ is a prime filter in the distributive lattice $\operatorname{Sub}_{\mathcal{C}}(X)$; these comprise the objects of a category $\mathcal{P} \mathcal{F}_{\mathcal{C}}$ of prime filters in $\mathcal{C}$ defined similarly to $\mathcal{U F}_{\mathcal{C}}$. Endowing $\mathcal{P} \mathcal{F}_{\mathcal{C}}$ with the obvious analogy of the topology of Proposition 41 yields [29]'s topos of existential types. No universal property is described, but the formula (3.6) appears on p.11 of ibid.

Makkai's topos of types from [38] is a topos of sheaves on the same category $\mathcal{P} \mathcal{F}_{\mathcal{C}}$, but for a different topology, and he exhibits it as the "classifying primegenerated topos for $\mathfrak{p}$-models of $\mathcal{C}$ ". As we have already said, a Grothendieck topos $\mathcal{E}$ is prime-generated if each subobject lattice is completely distributive algebraic, while a pretopos morphism $F: \mathcal{C} \rightarrow \mathcal{E}$ into a prime-generated topos is said to be a $\mathfrak{p}$-model if for every prime filter $\mathfrak{p}$ on $\operatorname{Sub}_{\mathcal{C}}(X)$ and every $f: X \rightarrow Y$ we have $\exists_{f}\left(\bigcap_{A \in \mathfrak{p}} F A\right)=\bigcap_{A \in \mathfrak{p}} F\left(\exists_{f} A\right)$ in $\operatorname{Sub}_{\mathcal{E}}(F Y)$. The classifying property of the topos of types $\tau(\mathcal{C})$ is given by equivalences

$$
\operatorname{PGTop}(\mathcal{E}, \tau(\mathcal{C})) \simeq \operatorname{pPretop}(\mathcal{C}, \mathcal{E})
$$

where to the left we have the category of geometric morphisms between primegenerated toposes whose inverse image functors preserve all intersections, and to the right we have the category of $\mathfrak{p}$-models. In establishing this equivalence, the formula (3.6) again appears; see the bottom of p.164 of ibid. In model-theoretic terms, the condition of being a $\mathfrak{p}$-model is a saturation condition; Makkai states this already in [38], and the point is followed up in [10], and exploited in, among other places, [43, 15]. 
The other main "topos of types" in the literature is Pitts' topos of filters $\Phi(\mathcal{C})$ of a pretopos $\mathcal{C}$. Introduced in [44], this is the topos of sheaves on the category $\mathcal{F}_{\mathcal{C}}$ of all - not necessarily prime - filters of subobjects, for the topology whose covers are the finite jointly epimorphic families. The universal property of $\Phi(\mathcal{C})$ was given in [37] by analogy with $\tau(\mathcal{C})$ : it is the "classifying completely distributive topos for $\mathfrak{f}$-models of $\mathcal{C}$ ". Here, a completely distributive topos is one whose subobject lattices are completely distributive, and an $\mathfrak{f}$-model is like a $\mathfrak{p}$-model, but with arbitrary filters replacing prime ones.

We conclude this discussion by comparing the universal characterisation (6.11) of Makkai's topos of types and our Theorem 42. To the left of the equivalence, our theorem replaces "prime-generated" by "locally connected" and moreover relaxes the condition of intersection-preservation on morphisms. What permits this relaxation is the fact that we only care about intersections of coproduct summands, and any inverse image functor between locally connected toposes preserves these. To the right of the equivalence, we drop the $\mathfrak{p}$-model condition. This is to do with the fact that our choice of topology is analogous to Joyal and Reyes' [29] rather than Makkai's [38]. If one modifies Makkai's topos of types to use Joyal and Reyes' topology, then one can also drop the p-model condition; however, the result is then no longer a prime-generated topos, and so it is unclear what an appropriate universal property would be. The final difference we note is that Makkai's equivalence works for arbitrary pretoposes $\mathcal{C}$, while ours works only for De Morgan pretoposes; this extra condition seems to be necessary to ensure that the topos of sheaves we form is indeed locally connected.

Asides from these technical distinctions, we would raise one further point. In this paper, we have striven to make the constructions we give as unavoidable as possible. The category $\mathcal{U F}_{\mathcal{C}}$ is forced upon us once we are interested in finitecoproduct-preserving functors out of $\mathcal{C}$; adding finite-limit-preservation leads us to consider also flatness; and finally, once we add regular-epimorphism-preservation, we are led inevitably to the given topology on $\mathcal{U} \mathcal{F}_{\mathcal{C}}$. Everything else is a matter of making the details match $\mathrm{up}^{2}$. In future work, we intend to see whether our main results can be adapted to the prime filter setting, and if, on doing so, they provide a treatment of Makkai's topos of types in the same spirit.

\section{REFERENCES}

[1] BARR, M. Exact categories. In Exact categories and categories of sheaves, vol. 236 of Lecture Notes in Mathematics. Springer, 1971, pp. 1-120.

[2] BARR, M., AND MAKKAI, M. On representations of Grothendieck toposes. Canadian Journal of Mathematics. Journal Canadien de Mathématiques 39 (1987), 168-221.

[3] Barr, M., And PARÉ, R. Molecular toposes. Journal of Pure and Applied Algebra 17 (1980), 127-152.

[4] BÉnabou, J. Introduction to bicategories. In Reports of the Midwest Category Seminar, vol. 47 of Lecture Notes in Mathematics. Springer, 1967, pp. 1-77.

[5] Blass, A. Orderings of Ultrafilters. PhD thesis, Harvard University, 1970.

[6] Blass, A. Two closed categories of filters. Fundamenta Mathematicae 94 (1977), 129-143.

\footnotetext{
${ }^{2}$ Though at this stage we have no satisfactory explanation for the requirement of De Morganness.
} 
[7] Blass, A. Ultrafilters: where topological dynamics = algebra = combinatorics. Topology Proceedings 18 (1993), 33-56.

[8] Borceux, F. Handbook of categorical algebra 1, vol. 50 of Encyclopedia of Mathematics and its Applications. Cambridge University Press, 1994.

[9] Börger, R. A characterization of the ultrafilter monad. Seminarberichte 6, Fernuniversität Hagen, 1980.

[10] Butz, C. Saturated models of intuitionistic theories. Annals of Pure and Applied Logic 129 (2004), 245-275.

[11] Caramello, O. De Morgan classifying toposes. Advances in Mathematics 222 (2009), $2117-2144$.

[12] Carboni, A., Lack, S., and Walters, R. F. C. Introduction to extensive and distributive categories. Journal of Pure and Applied Algebra 84 (1993), 145-158.

[13] Coumans, D. Generalising canonical extension to the categorical setting. Annals of Pure and Applied Logic 163 (2012), 1940-1961.

[14] Deligne, P. Theorie des topos et cohomologie étale des schemas (SGA 4), vol. 269 of Lecture Notes in Mathematics. Springer, 1972.

[15] Eliasson, J. Ultrasheaves. PhD thesis, University of Uppsala, 2003.

[16] Fiore, M., Gambino, N., Hyland, M., and Winskel, G. The cartesian closed bicategory of generalised species of structures. Journal of the London Mathematical Society 77 (2008), 203-220.

[17] Frayne, T., Morel, A. C., And Scott, D. S. Reduced direct products. Fundamenta Mathematicae 51 (1962/1963), 195-228.

[18] Funk, J. The locally connected coclosure of a Grothendieck topos. Journal of Pure and Applied Algebra 137 (1999), 17-27.

[19] Gabriel, P., And Zisman, M. Calculus of fractions and homotopy theory, vol. 35 of Ergebnisse der Mathematik und ihrer Grenzgebiete. Springer, 1967.

[20] Garner, R. Lawvere theories, finitary monads and Cauchy-completion. Journal of Pure and Applied Algebra 218 (2014), 1973-1988.

[21] Gehrke, M., And Jónsson, B. Bounded distributive lattices with operators. Mathematica Japonica 40, 2 (1994), 207-215.

[22] Grätzer, G., And Schmidt, E. T. On a problem of M. H. Stone. Acta Mathematica Academiae Scientiarum Hungaricae 8 (1957), 455-460.

[23] Halpern, J. D., And LÉvy, A. The Boolean prime ideal theorem does not imply the axiom of choice. In Axiomatic Set Theory (Proc. Sympos. Pure Math., Vol. XIII, Part I, Univ. California, Los Angeles, Calif., 1967) (1971), Amer. Math. Soc., Providence, R.I., pp. 83-134.

[24] HARnik, V. Model theory vs. categorical logic: two approaches to pretopos completion (a.k.a. $T^{\mathrm{eq}}$ ). In Models, logics, and higher-dimensional categories, vol. 53 of CRM Proc. Lecture Notes. Amer. Math. Soc., Providence, RI, 2011, pp. 79-106.

[25] Johnstone, P. T. Sketches of an elephant: a topos theory compendium. Vol. 1, vol. 43 of Oxford Logic Guides. Oxford University Press, 2002.

[26] Johnstone, P. T. Sketches of an elephant: a topos theory compendium. Vol. 2, vol. 44 of Oxford Logic Guides. Oxford University Press, 2002.

[27] Jónsson, B., And TARski, A. Boolean algebras with operators. I. American Journal of Mathematics 73 (1951), 891-939.

[28] Joyal, A. Foncteurs analytiques et espèces de structures. In Combinatoire énumérative (Montreal, 1985), vol. 1234 of Lecture Notes in Mathematics. Springer, 1986, pp. 126-159.

[29] Joyal, A., And Reyes, G. E. Forcing and generic models in categorical logic. Unpublished preprint, 1978.

[30] $\mathrm{KaT}^{\vee}$ etov, M. Products of filters. Commentationes Mathematicae Universitatis Carolinae 9 (1968), 173-189.

[31] Kelly, G. M. Basic concepts of enriched category theory, vol. 64 of London Mathematical Society Lecture Note Series. Cambridge University Press, 1982. Republished as: Reprints in Theory and Applications of Categories 10 (2005). 
[32] Kennison, J. F., And Gildenhuys, D. Equational completion, model induced triples and pro-objects. Journal of Pure and Applied Algebra 1 (1971), 317-346.

[33] Koubek, V., And Reiterman, J. On the category of filters. Commentationes Mathematicae Universitatis Carolinae 11 (1970), 19-29.

[34] Leinster, T. Codensity and the ultrafilter monad. Theory and Applications of Categories 28 (2013), 332-370.

[35] LuRIE, J. Ultracategories. Preprint, available at https://www.math.ias.edu/ lurie/ papers/Conceptual.pdf., 2019.

[36] Mac Lane, S., And MoerdiJk, I. Sheaves in geometry and logic. Universitext. Springer, 1992.

[37] Magnan, F. Le topos des types et le topos des filtres en logique categorique. ProQuest LLC, Ann Arbor, MI, 2000. Thesis (Ph.D.)-Universite de Montreal (Canada).

[38] Makkai, M. The topos of types. In Logic Year 1979-80 (Proc. Seminars and Conf. Math. Logic, Univ. Connecticut, Storrs, Conn., 1979/80), vol. 859 of Lecture Notes in Math. Springer, 1981, pp. 157-201.

[39] Makkai, M. Full continuous embeddings of toposes. Transactions of the American Mathematical Society 269 (1982), 167-196.

[40] Makkai, M. Stone duality for first order logic. Advances in Mathematics 65 (1987), 97-170.

[41] Makkai, M. Duality and definability in first order logic. Memoirs of the American Mathematical Society 105 (1993).

[42] Makkai, M., And Reyes, G. E. First order categorical logic, vol. 611 of Lecture Notes in Mathematics. Springer, 1977.

[43] MoerdiJk, I. A model for intuitionistic non-standard arithmetic. Annals of Pure and Applied Logic 73 (1995), 37-51.

[44] Pitrs, A. M. An application of open maps to categorical logic. Journal of Pure and Applied Algebra 29 (1983), 313-326.

[45] Street, R. Fibrations in bicategories. Cahiers de Topologie et Geométrie Différentielle Catégoriques 21 (1980), 111-160.

[46] Troelstra, A. S., And van Dalen, D. Constructivism in mathematics. Vol. I, vol. 121 of Studies in Logic and the Foundations of Mathematics. North-Holland Publishing Co., Amsterdam, 1988. An introduction.

[47] Walters, R. F. C. Sheaves and Cauchy-complete categories. Cahiers de Topologie et Geométrie Différentielle Catégoriques 22 (1981), 283-286.

Centre of Australian Category Theory, Macquarie University, NSW 2109 , Australia

E-mail address: richard.garner@mq.edu.au 